\title{
Nuclear dengue virus NS5 antagonizes expression of PAF1- dependent immune response genes
}

\author{
Marine J. Petit ${ }^{a, b}$, Matthew W. Kenaston ${ }^{a, \#}$, Ariana A. Nagainis ${ }^{a, b, \#}$, and Priya S. Shaha, ${ }^{a}$ \\ ${ }^{a}$ Department of Microbiology and Molecular Genetics \\ ${ }^{\mathrm{b}}$ Department of Chemical Engineering \\ \# These authors contributed equally \\ *Correspondence: prsshah@ucdavis.edu
}

\section{Abstract}

Dengue virus (DENV) disruption of the innate immune response is critical to establish infection. DENV non-structural protein 5 (NS5) plays a central role in this disruption, such as antagonism of STAT2. We recently found that DENV serotype 2 (DENV2) NS5 interacts with Polymerase associated factor 1 complex (PAF1C). The primary members of PAF1C are PAF1, LEO1, CTR9 and CDC73. This nuclear complex is an emerging player in the immune response. It promotes the expression of many genes, including genes related to the antiviral, antimicrobial and inflammatory responses, through close association with the chromatin of these genes. Our previous work demonstrated that NS5 antagonizes PAF1C recruitment to immune response genes. However, it remains unknown if NS5 antagonism of PAF1C is complementary to its antagonism of STAT2. Here, we show that knockout of PAF1 enhances DENV2 infectious virion production. By comparing gene expression profiles in PAF1 and STAT2 knockout cells, we find that PAF1 is necessary to express immune response genes that are STAT2-independent. Finally, we mapped the viral determinants for the NS5-PAF1C protein interaction. We found that NS5 nuclear localization and the C-terminal region of the methyltransferase domain are required for its interaction with PAF1C. Mutation of these regions rescued the expression of PAF1-dependent immune response genes that are antagonized by NS5. In sum, our results support a role for PAF1C in restricting DENV2 replication that NS5 antagonizes through its protein interaction with PAF1C. 


\section{Author summary}

Dengue virus (DENV) is a pathogen that infects nearly 400 million people a year and thus represents a major challenge for public health. Productive infection by DENV relies on the effective evasion of intrinsic antiviral defenses and is often accomplished through virus-host protein interactions. Here, we investigate the recently discovered interaction between DENV nonstructural protein 5 (NS5) and the transcriptional regulator Polymerase associated factor 1 complex (PAF1C). Our work demonstrates PAF1C member PAF1 acts as an antiviral factor and inhibits DENV replication. In parallel, we identified immune response genes involved in intrinsic antiviral defense that depend on PAF1 for expression. We further identified the regions of NS5 required for the protein interaction with PAF1C. Breaking the NS5-PAF1C protein interaction restores the expression of PAF1-dependent immune response genes. Together, our work establishes the antiviral role of PAF1C in DENV infection and NS5 antagonism of PAF1dependent gene expression through a virus-host protein interaction.

\section{Introduction}

Dengue virus (DENV) is a major source of human disease and is responsible for nearly 400 million infections annually (1). DENV belongs to the Flavivirus genus of viruses, which are positive sense, capped single-stranded RNA viruses that rely on multifunctional proteins to replicate. Flavivirus non-structural protein 5 (NS5) functions as both the RNA-dependent RNA polymerase (RdRp) (2) and the methyltransferase (MTase) (3-5). Genome replication is the main enzymatic activity of NS5 and occurs on the cytosolic side of ER-associated viral replication factories. In addition to playing a central role in genome replication, NS5 is a key player in disabling the innate immune response during DENV infection. Importantly, host protein interactions with NS5 are critical to its role in immune evasion. DENV NS5 binds human STAT2 and targets it for ubiquitin-mediated proteasomal degradation. This inhibits the expression of interferon-stimulated genes (ISGs) through interferon type 1 (IFN-I) signaling (6).

Interestingly, NS5 also has a nuclear role. NS5 localization to the nucleus during infection has been known for over 25 years (7) and observed by several other groups independently (8-11). DENV mutants that retain some NS5 nuclear localization are viable and can even replicate at near-wild-type (WT) levels. On the other hand, mutants that essentially eliminate NS5 nuclear localization are lethal (8-10). These defects in replication are not due to decreased intrinsic 
enzyme activity of NS5 $(8,9)$. We and others have shown that NS5 interacts with many nuclear proteins (12-14) and inhibits antiviral gene expression, potentially through these interactions $(13,14)$. Thus, some nuclear localization of NS5 is essential for replication and rewiring host gene expression, but the mechanisms by which nuclear DENV NS5 accomplishes these tasks are not completely understood.

Using a comprehensive global proteomics approach that defined the DENV-host protein interaction landscape, we recently found DENV serotype 2 (DENV2) NS5 interacts with and antagonizes Polymerase associated factor 1 complex (PAF1C) (14). This nuclear complex, whose primary members are PAF1, LEO1, CTR9 and CDC73, is an emerging player in the immune response. The complex generally promotes the expression of many genes, including genes related to the antiviral, antimicrobial and inflammatory responses $(15,16)$. We previously showed that NS5 antagonizes PAF1C by inhibiting its recruitment to immune response genes (14). However, it is currently unknown if NS5 inhibits expression of immune response genes distinct from STAT2-dependent ISGs through this protein interaction. Such antagonism may also explain a possible synergistic role for nuclear NS5. Yet, these mechanistic details have not been explored.

Here, we investigate if NS5 antagonism of PAF1C may be complementary to the antagonism of STAT2. We find that PAF1 restricts DENV2 infectious virion production. We also find that PAF1 is required for the expression of genes that are primarily STAT2-independent following innate immune stimulation. We further mapped the viral determinants of the NS5-PAF1C interaction using affinity purification and immunoblot of NS5 mutants to show that NS5 nuclear localization and the C-terminal region of the MTase domain are required for its interaction with PAF1C. NS5 mutants with reduced binding to PAF1C also have reduced antagonism of PAF1-dependent and STAT2-independent genes. Taken together, PAF1C-mediated expression of immune response genes is STAT2-independent and breaking the NS5-PAF1C interaction rescues PAF1-dependent gene expression.

\section{Results}

\section{PAF1C member PAF1 restricts DENV2 replication}

PAF1C is an emergent antiviral factor in mammals $(15,16)$. We previously showed that PAF1C restricts DENV2 replication through the measurement of antigen-positive cells (14). To determine 
the impact of PAF1C on the full viral replication cycle, we measured infectious virion production in PAF1 knockout (PAF1 KO) A549 cells at several time points. Since the entire complex can be destabilized with depletion of PAF1 only (17), we chose this member as a target for knockout. Generating PAF1 KO cells required isolating single clones. Therefore, we generated PAF1 rescue cells to control for clonal and CRISPR/Cas9 off-target effects (Fig 1A). Infectious virions increased approximately 3 -fold at 48 and 72 hours post-infection in PAF1 KO cells (Fig 1B). This replication advantage in PAF1 KO cells was significant $(p \leq 0.05)$ but disappeared at 96 hours post-infection. Based on these results, we conclude that PAF1 restricts DENV2 infectious virion production in human cells.
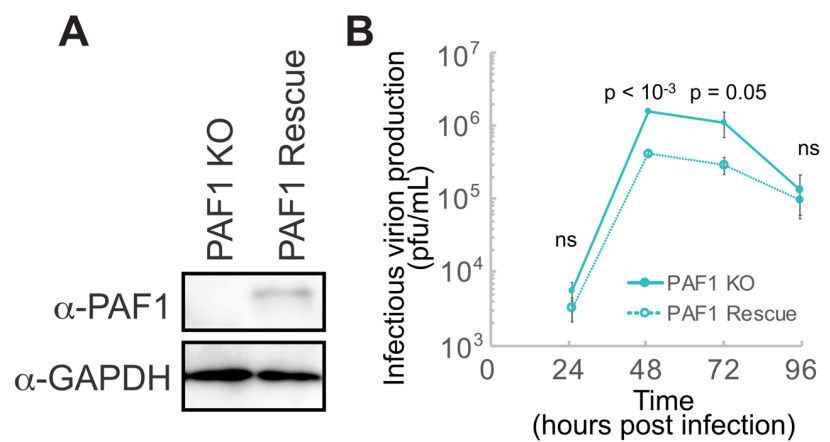

\section{Fig 1. PAF1 restricts DENV2 infectious virion production}

(A) Immunoblot analysis of PAF1 expression in PAF1 KO and rescue A549 cells. GAPDH is a loading control. (B) PAF1 $\mathrm{KO}$ and rescue cells were infected with DENV2 at MOI 0.1. Supernatants were harvested at the indicated times and infectious virion concentration was measured by limiting dilution plaque assay. Data from three independent biological replicates are plotted as mean values $+/$ - standard deviation. $P$ values were calculated using a paired, one-tailed Student's t-test. Abbreviations: plaque forming units (pfu), not statistically significant (ns).

\section{PAF1 is required for the expression of STAT2-independent genes}

PAF1C regulates the expression of stress response genes (14), including ISGs and inflammatory genes $(15,16)$. Since NS5 interacts with STAT2 and PAF1C, we hypothesized that PAF1C could play a unique role upstream or independent of STAT2-mediated IFN-I signaling. For this reason, we activated the immune response using a three-hour treatment with poly(l:C), a dsRNA mimic that can stimulate RIG-I, MDA5 and TLR3 signaling (18-21). Importantly, our treatment was long enough to induce IFNBI expression in A549 cells (log2 fold change 11.9, p adj < $10^{-19}$ ) (S1 Table) and could thus capture events upstream and downstream of IFN-I signaling. To distinguish the 
PAF1- and STAT2-dependent responses, we performed experiments in PAF1 and STAT2 KO and rescue cells (Fig 2A). We assessed data quality on a global level using principal component analysis (PCA) (Fig 2B). Strong clustering of parental A549, PAF1 rescue and STAT2 rescue cells suggests the effects observed are not a result of clonal or CRISPR/Cas9 off-target effects. Additionally, we show that PAF1 and STAT2 KO form distinct clusters for both poly(l:C) treated and control samples, suggesting that PAF1 and STAT2 have unique roles in basal and immune gene expression.

To gain additional insight into PAF1- and STAT2-mediated gene expression, we analyzed global changes using Gene Set Enrichment Analysis (GSEA) $(22,23)$. Several Reactome pathways were significantly enriched among PAF1 and STAT2 KO cells compared to parental A549 cells (Fig 2C, S1 and S2 Table). Distinct enriched pathways were observed for each genotype, corroborating our PCA results. As expected, pathways related to IFN-I signaling were significantly downregulated in STAT2 KO cells ( $p$ adj < 0.01). Extracellular matrix organization $(E C M)$ pathways were also significantly upregulated in STAT2 KO cells, indicating an additional role for STAT2 in repressing the expression of these genes ( $p$ adj < 0.01). Top downregulated categories in PAF1 KO cells were related to PAF1C roles in chromatin modification ( $p$ adj < 0.005 ) or regulation of TP53 and DNA double-strand break repair pathways ( $p$ adj $<0.1$ ). One significantly downregulated category, the DDX58-IFIH1 (RIG-I-MDA5) induction of IFN-I response (p adj < 0.05), is important for the production of IFN-I during DENV infection (24-29). Therefore, PAF1 may regulate the immune response upstream of IFN-I signaling.

Surprisingly, significantly upregulated pathways in PAF1 KO cells revealed several categories that may be pro-flaviviral in nature (Fig 2C). While generally thought of as a transcription elongation factor that promotes gene expression, PAF1C can repress expression in some contexts, such as genes with highly active super-enhancers in cancer cells (30). Given the context-dependent nature of PAF1C in promoting or repressing gene expression, we hypothesized that PAF1C-mediated repression of pro-flaviviral genes may contribute to PAF1C restriction of DENV2 replication. As such, we examined genes composing the upregulated pathways in PAF1 KO cells and found many were related to known flavivirus host dependency factors. To rigorously test if PAF1 was repressing the expression of pro-flaviviral factors, we performed GSEA on flavivirus host dependency factors identified through genetic screens (3136) (S3 Table). We found that PAF1 KO cells had significant upregulation of expression of these 
genes ( $p$ adj < 0.005) (S2 Fig). This effect is PAF1-specific as STAT2 KO cells did not show a significant change in expression for this group of genes.

We next explored the role of PAF1 in the expression of specific genes. We analyzed immune response genes (GO:0006955) whose expression was significantly induced in parental A549 cells following poly $(\mathrm{I}: \mathrm{C}$ ) treatment (log2 fold change > 0.5, p adj < 0.05) (S4 Table). This subset of genes allows the unbiased comparison of PAF1 and STAT2 KO cells to parental A549 cells in the context of the immune response. Both PAF1 and STAT2 KO resulted in the significant inhibition of expression of these immune response genes relative to parental A549 cells (Fig 2D). We also looked at genes that were significantly downregulated in PAF1 or STAT2 KO cells compared to A549 cells following poly(l:C) treatment (Fig 2E). While a few genes were significantly affected by both PAF1 and STAT2 KO, many genes displayed PAF1- and STAT2-specific responses. The overlap between the PAF1- and STAT2-dependent gene sets was significantly lower than expected by chance $(p<0.05)$. Unbiased k-means clustering formed unique clusters comprising PAF1- and STAT2-dependent genes, further underlining these differences. Our results indicate that PAF1 and STAT2 have distinct but complementary roles and might function in synergy following poly(l:C) stimulation. 
bioRxiv preprint doi: https://doi.org/10.1101/2021.04.06.438514; this version posted May 4, 2021. The copyright holder for this preprint (which

was not certified by peer review) is the author/funder, who has granted bioRxiv a license to display the preprint in perpetuity. It is made available under aCC-BY-NC-ND 4.0 International license.

A

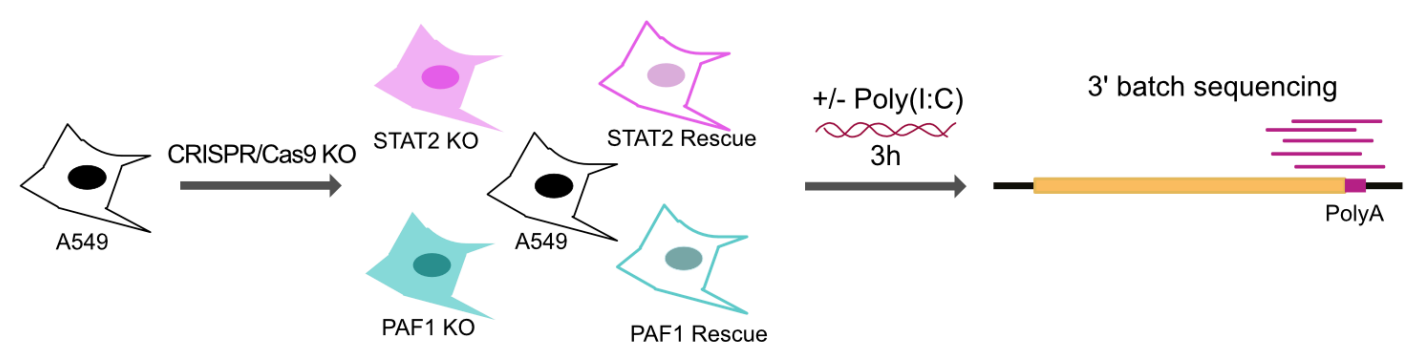

B

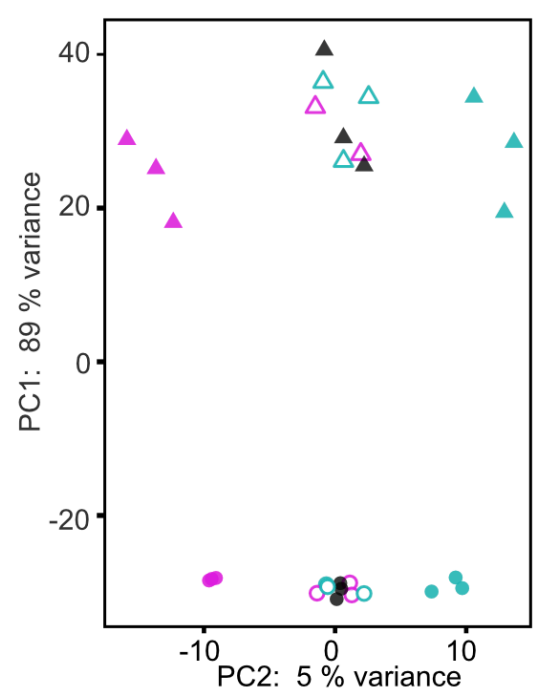

D

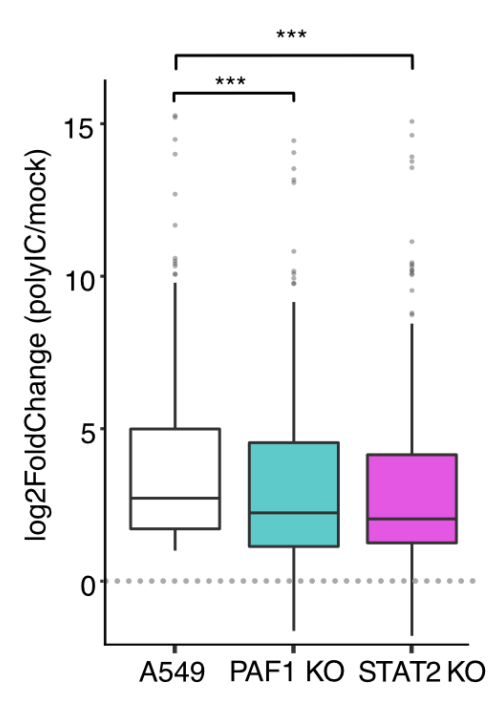

C

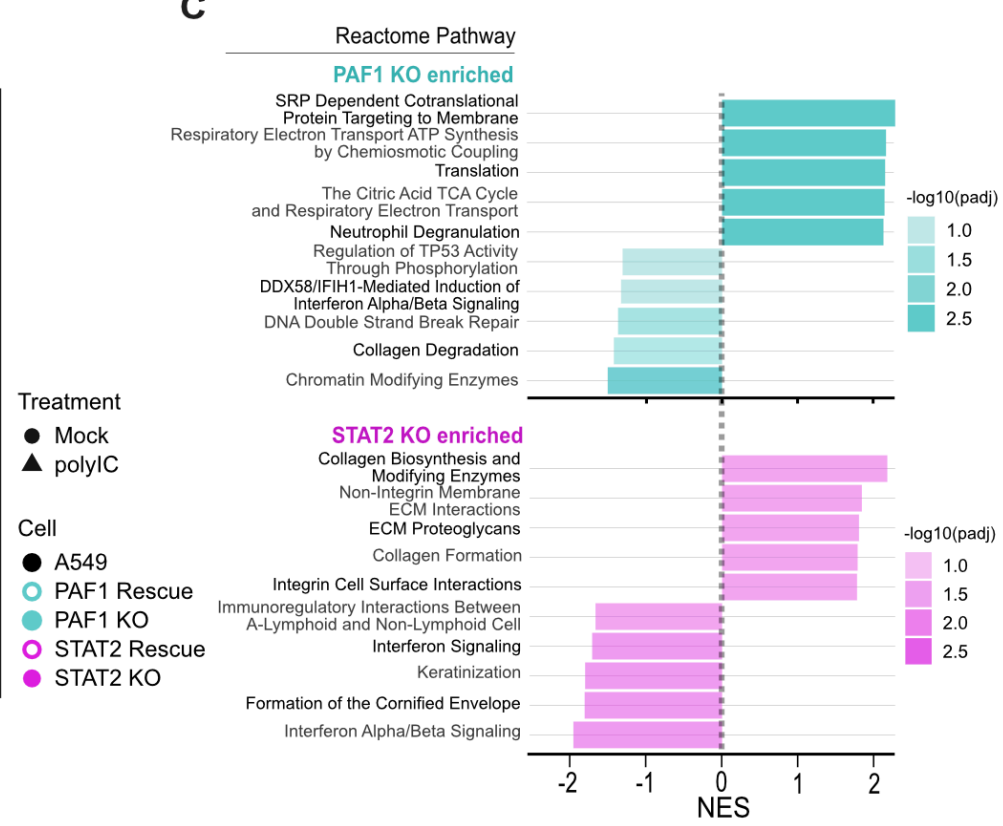

E

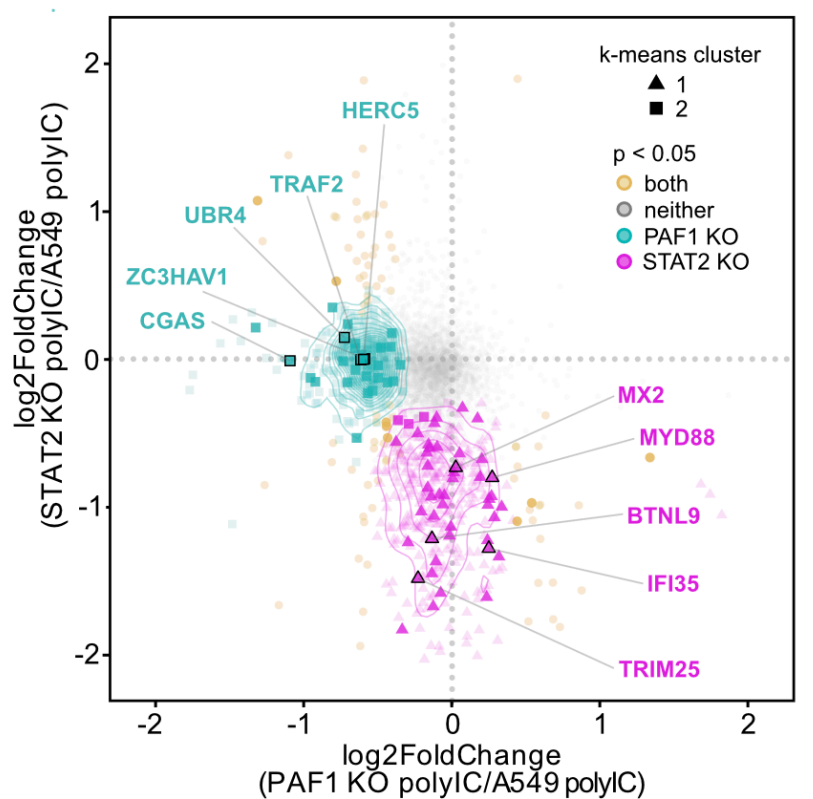




\section{Fig 2. PAF1 is required for expression of a subset genes activated by poly(I:C)}

(A) Parental A549, PAF1 and STAT2 KO, and rescue cells were stimulated with poly(I:C) for 3h and subjected to RNA-seq and DESeq2 differential gene expression analysis. Results are based on three independent biological replicates. (B) Principal component analysis performed on all samples showed a clear separation between principal components (PC) describing untreated/treated cells and cell genotype. (C) GSEA was performed on genes differentially expressed in $\mathrm{KO}$ cells compared to parental A549 cells following poly(l:C) treatment. Up to the top 5 positively and negatively enriched Reactome pathways were plotted for each comparison ( $p$ adj < 0.1). A full list of GSEA results is available in S2 Table. (D) Changes in gene expression caused by poly $(\mathrm{I}: \mathrm{C})$ treatment are shown for the subset of immune response genes (G0:0006955) significantly upregulated for poly(I:C)-treated parental A549 cells relative to mock-treated A549 cells (log2 fold change > 0.5, padj < 0.05). A Wilcoxon signed rank test with Bonferroni correction was performed to identify significant changes caused by PAF1 or STAT2 KO. (E) All genes significantly upregulated for poly(l:C)-treated parental A549 cells relative to mock-treated A549 cells (log2 fold change > 0.5, padj < 0.05) were plotted based on log2 fold change of PAF1 and STAT2 KO cells relative to parental A549 cells following poly(I:C) treatment. Parental A549 cells were used as a normalization so that it was identical for both comparisons. Unsupervised Kmeans clustering was also performed to identify genes with similar behavior (triangles, circles and squares). $P$ values were adjusted for false discovery rate using the Benjamini Hochberg method. Significant changes in gene expression are plotted for PAF1 KO (cyan), STAT2 KO (magenta), both (yellow) or neither (grey). Immune response genes (GO:0006955) are highlight with larger markers and opaque coloring.

\section{Nuclear localization is required for the NS5-PAF1 interaction}

To establish if the NS5 interaction with PAF1C is required for antagonism, we set out to identify the viral determinants of the interaction. Given the strong nuclear localization of NS5 during infection $(8,10,37)$, we tested if this nuclear localization is required for its interaction with PAF1. We generated NS5 mutants for the central and C-terminal nuclear localization signals (NLSs) alone and together (Fig 3A) (8,9). We used immunofluorescence confocal microscopy to determine the subcellular localization of these NS5 mutants. Compared to WT NS5, the single NLS mutants (NS5 central and NS5c-term) showed some decrease in nuclear signal. Only the double mutant (NS5 $2 \times N L S$ ) fully abrogated nuclear localization and colocalization with PAF1 (Fig 3B). 
We next determined if nuclear localization of NS5 is required for its interaction with PAF1C using affinity purification and immunoblot of WT NS5 and the NS5 $5_{2 \times N L S}$ mutant. As expected, we observed an interaction between WT NS5 and PAF1. This interaction was lost for NS5 $2 \times N L s$ (Fig 3C). Moreover, while we observed PAF1 and WT NS5 in cytoplasmic and nuclear compartments using biochemical fractionation, we only found NS5 to interact with PAF1 in the nuclear fraction (S3 Fig). Together, our results show that NS5 nuclear localization is required for its interaction with PAF1.

A

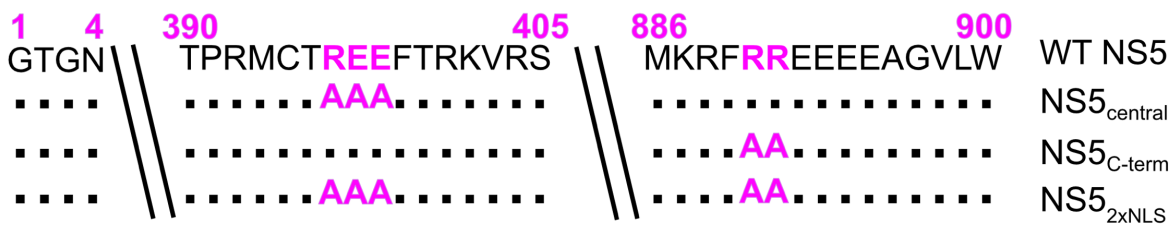

B
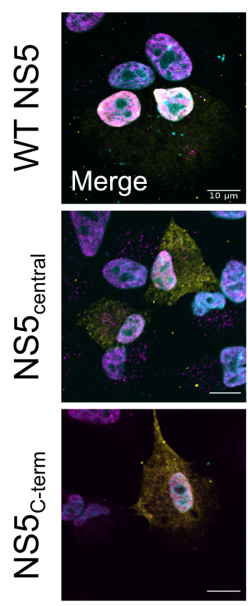

(

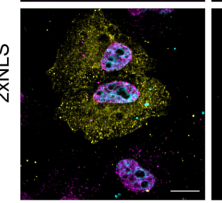

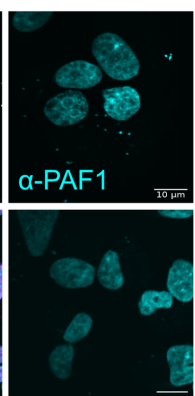
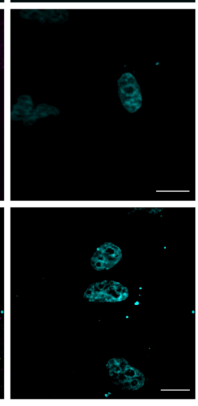
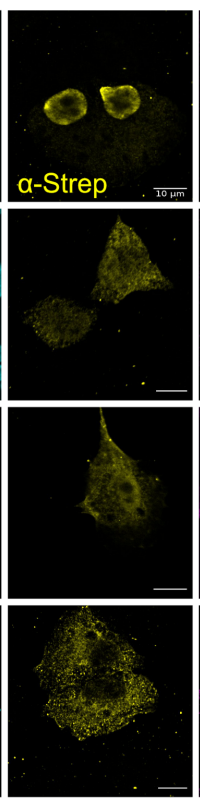
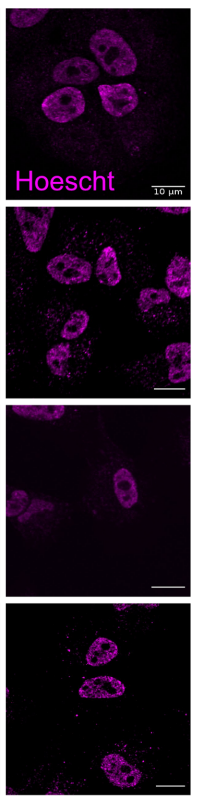

C

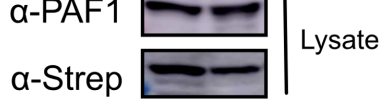

a-PAF1

a-Strep

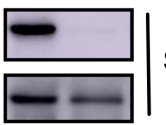
Strep-AP

Fig 3. Nuclear localization is required for NS5-PAF1C interaction

(A) NLS mutants, including single mutant NS5 ${ }_{\text {central }}$ (REE396-398AAA) and NS5c-term (RR890891AA) and the double mutant NS5 $2 \times$ NLS (REE396-398AAA and RR890-891AA). (B) Subcellular localization of 2xStrep II tagged NS5s (yellow) and colocalization with PAF1 (cyan) was determined by immunostaining and confocal microscopy. Nuclei were stained with Hoechst (magenta). Scale bar represents $10 \mu \mathrm{m}$. (C) The protein interaction between NS5 $2 \times N$ Ls and PAF1 was tested biochemically. Plasmids encoding NS5s and GFP were transfected and affinity purified in HEK293T cells via a 2xStrep II tag. Immunoblot analysis of lysate and purified (Strep-AP) 
fractions was performed against PAF1, Strep and GAPDH (loading/negative control). Abbreviations: Strep-affinity purified (Strep-AP).

\section{A highly conserved region of the C-terminus of the NS5 MTase domain is required for the interaction with PAF1C}

While the nuclear localization of NS5 is required for the NS5-PAF1 interaction, the viral molecular determinants of the interaction surface may be different. To identify these determinants, we generated a series of $\mathrm{N}$ - and C-terminally truncated NS5 constructs (Fig 4A). Importantly, all constructs conserved the central NLS region necessary for some nuclear localization and interaction with PAF1. The subcellular localization of all our truncations was assayed by immunofluorescence confocal microscopy (Fig 4B). As expected, all truncations had some nuclear localization. We also observed that truncations missing the C-terminal NLS (T4/5/6/7) were less abundant in the nucleus, reenforcing the importance of both the central and C-terminal NLS for nuclear localization $(8,9)$.

To determine which region of NS5 interacts with PAF1, we used affinity purification and immunoblot. All truncations except T3 ( $\Delta 1-313)$ interacted with PAF1 (Fig 4C), meaning that NS5PAF1 interaction determinants are in the region separating T2 and T3 (amino acids 210-313). This region contains the $\mathrm{C}$-terminus of the MTase domain, the linker and the $\mathrm{N}$-terminus of the RdRp domain. We hypothesized that PAF1C interacts with the MTase region since PAF1C also interacts with host methyltransferases that use S-adenosyl-methionine as a methyl donor $(38,39)$. Therefore, we generated two new truncations to target this region, T2A $(\Delta 1-222)$ and T2B $(\Delta 1$ 251). Both truncations interacted with PAF1 (Fig 4D), suggesting that the PAF1-interacting region of NS5 resides between amino acids 251 and 265 of the MTase. 
A

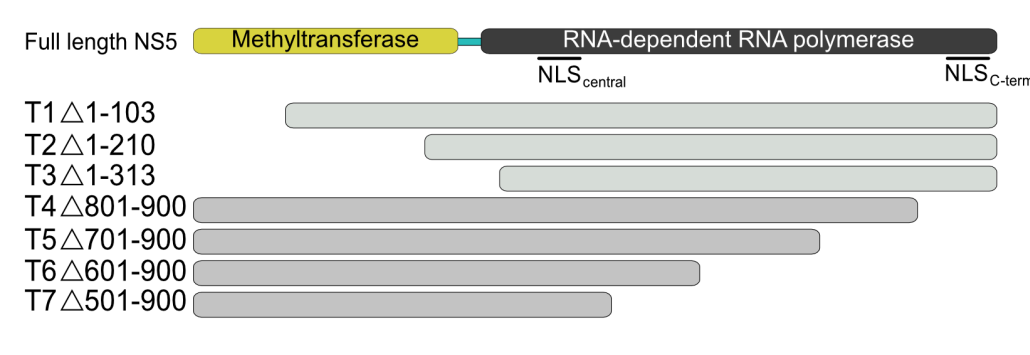

B

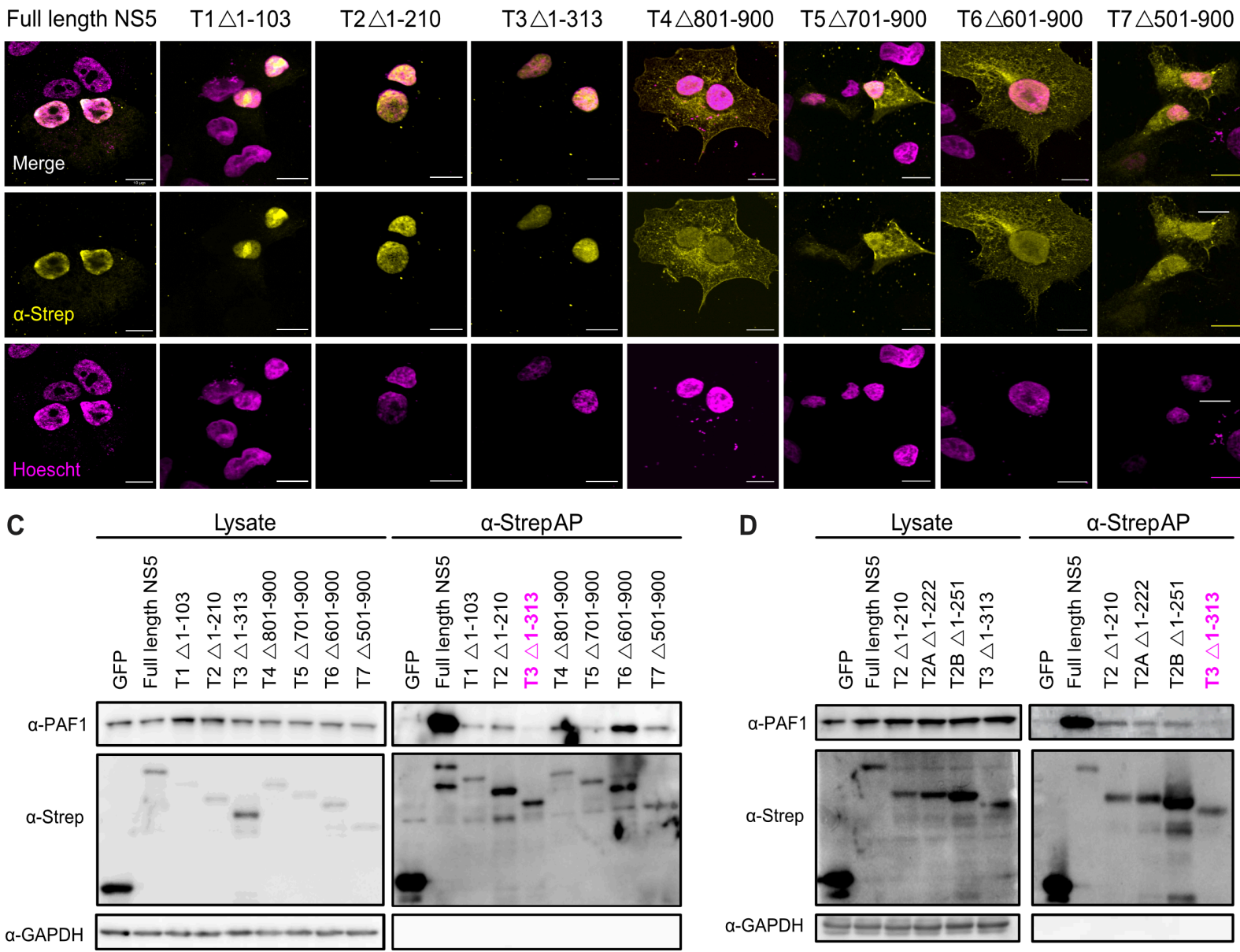

Fig 4. The $\mathrm{C}$-terminus Methyltransferase domain is responsible for interaction with PAF1C (A) NS5 truncations constructed to test NS5-PAF1 interaction. Each truncation corresponds to 100 amino acid deletions from the $\mathrm{N}-(\mathrm{T} 1, \mathrm{~T} 2, \mathrm{~T} 3)$ or C-terminus (T4, T5, T6, T7) of NS5. (B) Subcellular localization of 2xStrep II tagged truncations and NS5 full-length (yellow) and colocalization with PAF1 (cyan) were determined by immunostaining and confocal microscopy. Nuclei were stained with Hoechst (magenta). Scale bar represents $10 \mu \mathrm{m}$. (C) The protein interaction between NS5 truncations and PAF1 were tested biochemically. Plasmids encoding NS5 truncations, NS5 full-length and GFP were transfected and affinity purified in HEK293T cells via a 2xStrep II tag. 
Immunoblot analysis of lysate and purified (Strep-AP) fractions was performed against PAF1, Strep and GAPDH (loading/negative control). (D) Refined truncations in the T2-T3 region (T2, T2A, T2B and T3) were tested for an interaction with PAF1 biochemically. Purification and immunoblot analysis were conducted as in (C). Abbreviations: Strep-affinity purified (Strep-AP).

Conservation could help to identify specific amino acids of NS5 critical for its interaction with PAF1C. We and others previously observed the conservation of the NS5-PAF1C interaction across mosquito-borne flaviviruses $(14,40)$. We further sought to determine if this interaction was conserved amongst additional flaviviruses, including tick-borne flaviviruses. We tested different flavivirus NS5 proteins for subcellular localization and found that all had some nuclear localization, making an interaction with PAF1C possible (S4 Fig). Using affinity purification and immunoblot, we found all NS5s tested interacted with PAF1, including tick-borne encephalitis virus (TBEV), Langat virus (LGTV) and Saint-Louis Encephalitis virus (SLEV) (Fig 5A).

Alignment of the NS5 protein sequences between amino acids 251 and 265 revealed a highly conserved stretch of six amino acids that could be responsible for the interaction with PAF1C (Fig $5 \mathrm{C})$. This region precedes the pivot region of the inter-domain linker important for NS5 folding $(41,42)$ (Fig 5B). We performed site-directed mutagenesis on these six amino acids, creating two new NS5 mutants, LGS258AAA (NS5 localized predominantly in the nucleus (S4 Fig), they failed to interact with all four principal components of PAF1C (PAF1, LEO1, CDC73 and CTR9) (Fig 5D). These mutants still bound STAT2, albeit at a reduced level. In summary, residues in the C-terminus of the NS5 MTase domain are required for the NS5-PAF1C interaction. 
A

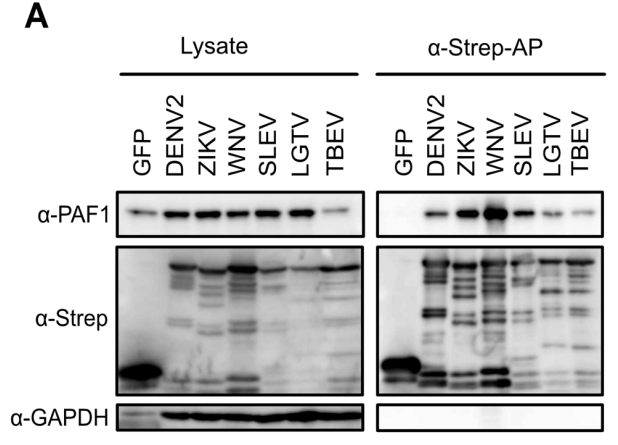

B

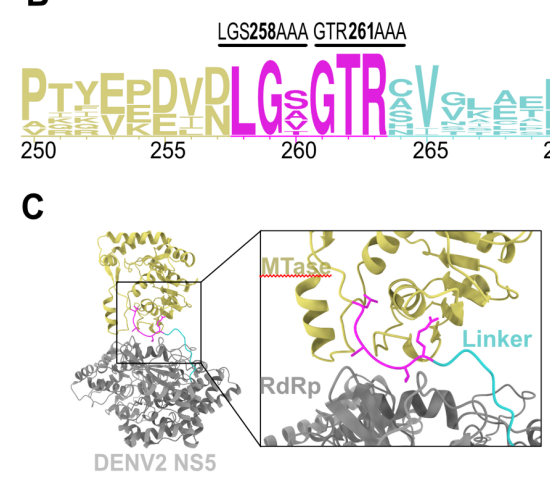

D

270
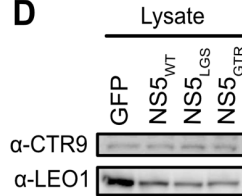

$\alpha-P A F 1-$

a-CDC73 $=\equiv$

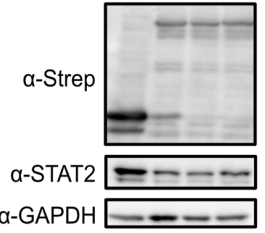

$\frac{\alpha \text {-Strep-AP }}{0.10^{5}}$

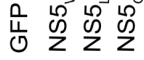

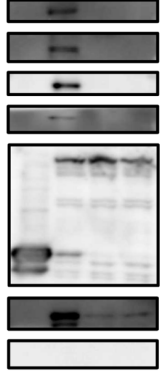

Fig 5. A highly conserved region of the MTase C-terminus is required for PAF1C binding

(A) The protein interaction between flavivirus NS5s and PAF1 was tested biochemically. Plasmids encoding NS5s and GFP were transfected and affinity purified in HEK293T cells via a 2xStrep II tag. Immunoblot analysis of lysate and purified (Strep-AP) fractions was performed against PAF1, Strep and GAPDH (loading/negative control). (B) DENV2 NS5 structure from PDB (5ZQK) with PAF1-interacting region highlighted (box). The PAF1-interacting region overlaps with the Cterminal end of the MTase (yellow) and the flexible linker domain (cyan), but not the RdRP (grey). The PAF1-interacting region includes a stretch of amino acids conserved in PAF1-interacting flaviviruses tested in (A). (C) Logo analysis of amino acid conservation for flavivirus NS5s from (A) in the PAF1-interacting region. The conserved stretch from amino acids 258-263 (magenta) was targeted for alanine scanning. Two different DENV2 NS5 mutants were constructed, LGS258-260AAA (NS5LGS) and GTR261-263AAA (NS5 ${ }_{G T R}$ ). (D) Mutants from (C) were tested for an interaction with PAF1C biochemically. Purification and immunoblot analysis were conducted as in (A). PAF1C complex members CTR9, LEO1, CDC73 and PAF1 were probed. STAT2 was used as a positive control for an NS5 interaction outside of the PAF1C-interacting region. Abbreviations: Zika virus (ZIKV), West Nile virus (WNV), Powassan virus (POWV), Langat virus (LGTV), tick-borne encephalitis virus (TBEV), Strep-affinity purified (Strep-AP).

The NS5-PAF1C interaction is required to antagonize PAF1-dependent expression of immune response genes.

We next determined if breaking the NS5-PAF1C interaction affects NS5 antagonism of immune gene expression. A549 cells expressing WT NS5 and our three mutants (NS5 LGS, NS5 $5_{\text {GTR }}$ and NS5 $5_{2 \times N L S}$ ) were subjected to 3' batch-tag RNA-seq following poly(I:C) stimulation (Fig 6A). Surprisingly, we observed significantly diminished induction of immune response genes in this 
experiment relative to knockout cells, despite using the same concentration of poly(l:C) and timepoint (S5A Fig, S5 Table). In exploring why this induction was dampened, we found strong induction of immune response genes in our mock samples from plasmid transfection alone, likely due to the use of lipofectamine 3000 (43). In fact, when comparing mock and poly(l:C)-treated plasmid-transfected cells, the induction of immune response genes in mock plasmid-transfected cells was more correlated with the induction of these same genes in A549 cells following poly(l:C) treatment (S5B Fig, S5 Table). We consequently focused our analysis on genes significantly induced (log2 fold change $>0.5$, p adj 0.05 ) under these more correlated stimulations.

We explored general trends using GSEA on genes differentially expressed following transfection of WT versus mutant NS5. GSEA revealed several interesting trends (Fig 6B, S2 Table). We observed significant positive enrichment of PAF1-dependent genes for NS5LGS and NS52×NLS compared to WT NS5 ( $\mathrm{p}$ adj < 0.1 and 0.05 , respectively), indicating these mutants fail to antagonize PAF1 as effectively as the WT. NS5 mutants also showed significant positive enrichment of genes related to IFN signaling compared to WT NS5 ( $p$ adj < 0.05). Importantly, there was also significant positive enrichment for genes related to DDX58-IFIH1 (RIG-I-MDA5) mediated induction of IFN-I for all three mutants ( $p$ adj < 0.05). The same pathway was significantly downregulated in our PAF1 KO cells (Fig 2C), supporting our hypothesis that the NS5-PAF1C protein interaction antagonizes expression of PAF1-dependent genes in this signaling pathway to enhance replication.

We next explored if the specific genes driving these general similarities are common or unique for each mutant. Volcano plots showed NS5 mutants predominantly displayed significantly increased expression of genes relative to WT NS5, rather than decreases (|log2 fold change| > 0.5, p adj < 0.05) (S6 Fig, S5 Table). By comparing the three NS5 mutants to each other using hierarchical clustering, we found that genes significantly perturbed by $N S 5_{2 \times N L S}$ formed a distinct cluster from NS5LGS and NS5GTR. These results suggest that the disruption of nuclear localization affects a broader set of genes than targeted disruption of the PAF1C interaction domain (Fig 6C). We further identified specific genes whose expression was rescued by all three mutants (e.g. IFIT2, DDX58), or specific to NS5 ${ }_{2 \times N L S}$ (e.g. CXCL10, IFNB1). 
A
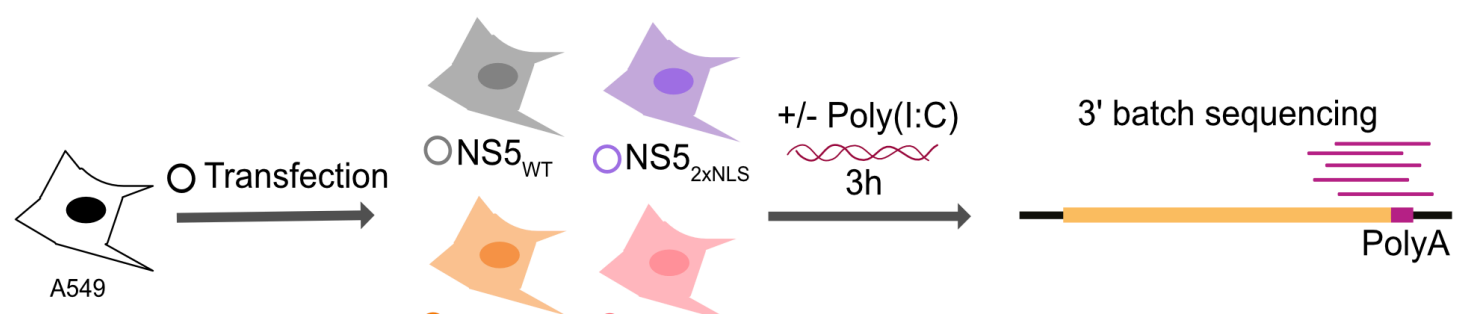

B

C

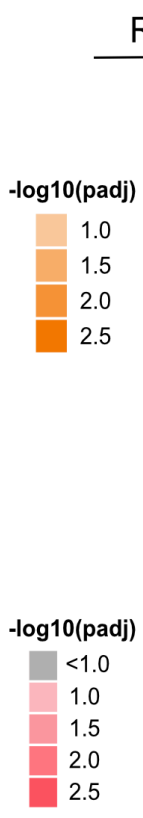

Reactome Pathway

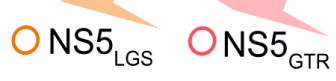

PolyA

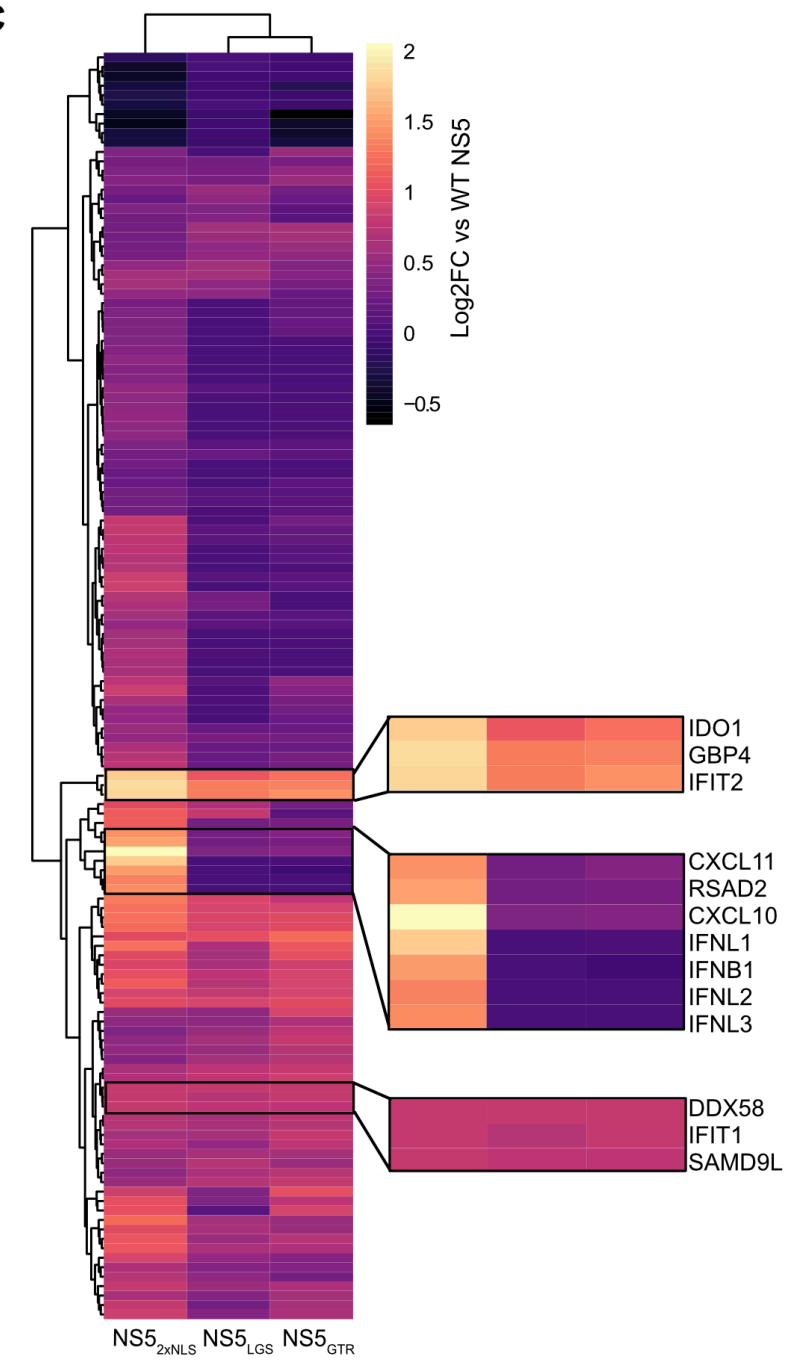

$-\log 10($ padj $)$

1.0

1.5

2.0

2.5

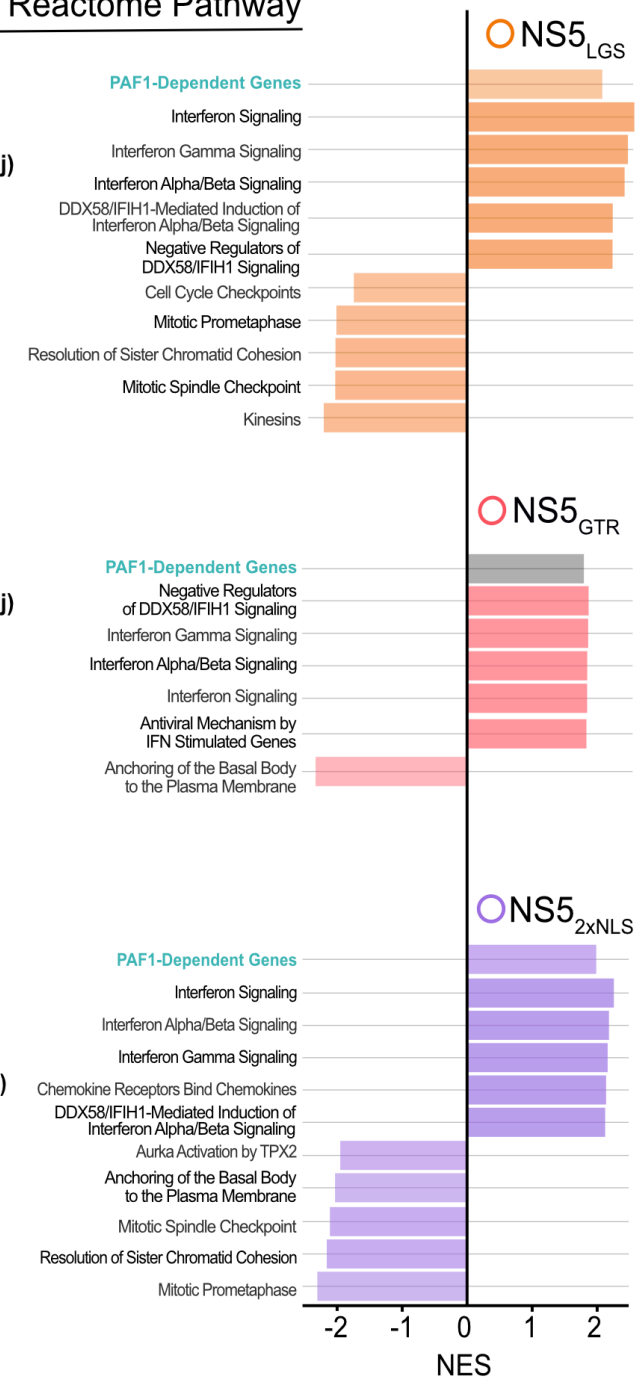

Fig 6. NS5 mutants have shared and distinct impacts on gene expression.

(A) Parental A549 cells were transfected with NS5wt (grey), NS52xNLs (purple), NS5LGs (orange) and NS5GTR (red) and GFP as a negative control. Transfected cells were stimulated with poly(I:C) for three hours and subjected to RNA-seq. Results are based on three independent biological 
replicates. (B) GSEA was conducted on genes differentially expressed by cells expressing WT versus mutant NS5s. Up to the top 5 positively and negatively enriched Reactome pathways were plotted for each comparison ( $p$ adj < 0.1). A full list of GSEA results is available in S2 Table. (C) Genes significantly upregulated in NS5 $2 \times$ NLS versus WT NS5 (log2 fold change $>0.5, p$ adj $<0.05$ ) were subjected to hierarchical clustering for each mutant and displayed as a heat map. Gene of interest are highlighted by zoom panels.

We finally sought to determine how gene expression rescued by NS5 mutants related to antagonism of PAF1. We compared the expression changes between mutant and WT NS5 to changes from PAF1 and STAT2 KO (Fig 7A, S1 and S5 Table). NS5 mutants rescued the expression of many genes inhibited by WT NS5, some of which were PAF1-dependent ( $p$ adj < 0.05). This subset of rescued PAF1-dependent genes was consistent between all three mutants, suggesting a crucial role for the NS5-PAF1C interaction in antagonizing the expression of these genes, specifically. These genes were also STAT2-independent (Fig 7B, S1 and S5 Table). Notably, for the immune response genes whose expression was rescued by NS5 mutants, there was a shift towards PAF1-dependency but not STAT2-dependency. This shift was statistically significant for all mutants $(p<0.05)$ and further underlines the complementary nature of NS5 antagonism of PAF1C in the nucleus. We further tested the subset of PAF1-dependent and STAT2-independent immune response genes using qRT-PCR (S7 Fig). Expression of these genes was rescued by NS5 mutants, though the effects were most consistent for the NS5 $5_{G T R}$ mutant. Thus, breaking the NS5-PAF1C interaction rescues the expression of PAF1-dependent genes. However, these mutants also rescue the expression of immune response genes that are PAF1- and STAT2-independent, suggesting additional mechanisms of immune antagonism by NS5. 
A
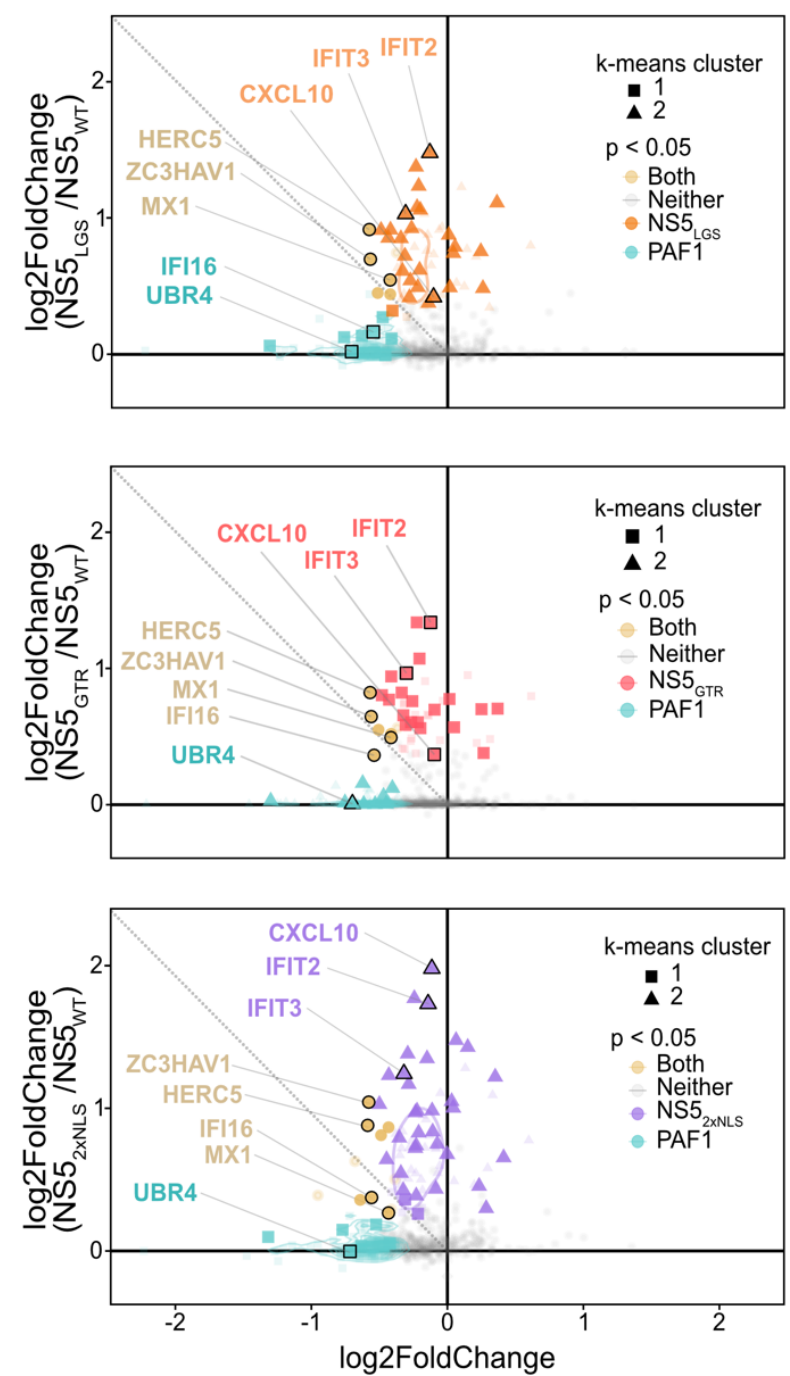

(PAF1 KO polylC/A549 polylC)
B STAT2
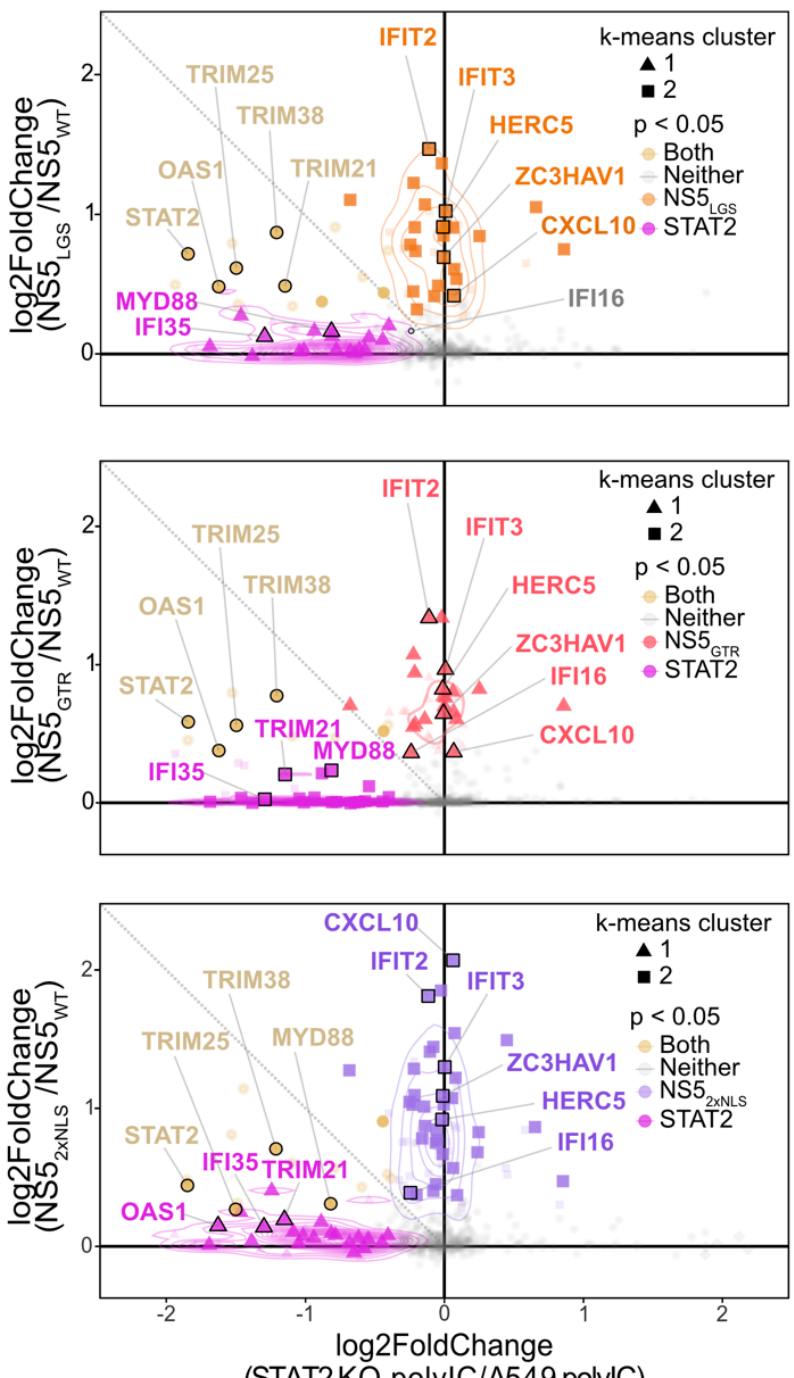

(STAT2KO polyIC/A549 polyIC)

Fig 7. NS5 mutants rescue expression of PAF1-dependent genes.

Relative gene expression was plotted as log2 fold change for (A) PAF1 KO versus parental A549 cells or (B) STAT2 KO versus parental A549 (same as Fig 2E), and NS5 mutant versus WT. Unsupervised K-means clustering was also performed to identify genes with similar behavior (triangles, circles and squares). $\mathrm{P}$ values were adjusted for false discovery rate using the Benjamini Hochberg method. Significant changes in gene expression are plotted for: PAF1 KO (cyan), both (yellow), neither (grey), NS5LGS (orange), NS5 $5_{G T R}$ (red) and NS5 $2 \times N L S$ (purple). Immune response genes (GO:0006955) are highlight with larger markers and opaque coloring. 


\section{Discussion}

Here, we explore gene expression mediated by PAF1C and antagonized by DENV2 NS5. We find that PAF1 restricts DENV2 infectious virion production and is required for the expression of poly(I:C)-induced genes that are STAT2-independent. We further map the viral determinants of the NS5-PAF1C interaction and show that both NS5 nuclear localization and residues in the Cterminus of the MTase domain are necessary for this interaction. Finally, we show that breaking the NS5-PAF1C interaction rescues expression of a subset of PAF1-dependent and STAT2independent immune response genes.

PAF1C has been viewed as a regulator of the host immune response and an antiviral host restriction factor (15). We previously showed that knockdown of PAF1C members increased DENV2 and Zika virus (ZIKV) replication measured by immunostaining for $E$ antigen (14). Here, we extend these results to demonstrate that PAF1 restricts DENV2 infectious virion production (Fig 1). This stands in contrast to a recent study with Japanese encephalitis virus (JEV), in which JEV appears to depend on PAF1 for viral protein production to some degree (40). Notably, JEV NS5 was also found to interact with PAF1C, suggesting that while the NS5-PAF1C interaction is broadly conserved among mosquito- and tick-borne flaviviruses (Fig 5A), the role of PAF1C in flavivirus replication may be virus or cell-type dependent.

Compared to previous studies, which focused on PAF1-mediated expression under basal conditions $(44,45)$ or following IFN-I treatment $(15)$, we identified PAF1-dependent immune response genes upstream of IFN-I signaling and/or independent of STAT2 (Fig 2). We also identified genes suppressed by PAF1, including flavivirus host dependency factors. Thus, PAF1 potentially restricts DENV2 replication through two independent mechanisms - up-egulation of the immune response and suppression of proviral genes. PAF1 is known to have contextdependent impacts on transcription elongation in mammalian cancer cell lines $(44,45)$ but appears to exclusively promote transcriptional elongation in primary mammalian cells (46). Thus, studying the PAF1C-mediated immune response in primary mammalian cells will be an important future step.

The innate immune response serves as a cell's first response to pathogen infection, which flavivirus NS proteins antagonize. NS5 is a prominent inhibitor of IFN-I responses, although 
mechanisms of antagonism are virus-dependent (6,47-49). Here, we demonstrate that NS5 interacts with PAF1C to inhibit the expression of PAF1-dependent immune response genes in a manner that is complementary to the established DENV NS5 antagonism of STAT2 (6). In particular, genes related to the DDX58-IFIH1 (RIG-I-MDA5) signaling pathway were found to be PAF1-dependent and antagonized by the NS5-PAF1C interaction. This could be advantageous to DENV given the role of both RIG-I and MDA5 in restricting DENV replication (24-29).

Our work also revealed novel insights into NS5 antagonism of IFN-I signaling. Compared to NS5 mutants, WT NS5 still inhibited genes related to IFN-I signaling despite not being able to degrade STAT2. Reduced binding of STAT2 by our NS5 $5_{G T R}$ and NS5LGS mutants may affect this process (Fig 5D). However, many of these genes are also STAT2-independent in A549 cells (S6 Fig). This could point to previously unknown mechanisms of NS5 antagonism of IFN-I signaling such as upstream DDX58-IFIH1 signaling. It will be valuable to study the effects of these mutations in the context of infection. Fortunately, mutations in this region have produced viable DENV2 mutants in the past (41).

Finally, our studies reveal a novel role of nuclear NS5 in antagonizing the host immune response. Previous studies with infectious NS5 NLS mutants found no defects in overcoming IFN-I restriction (8). However, mutants with complete loss of NS5 nuclear localization could not be studied using this approach because of their lethality. Here, we show that nuclear localization of NS5 is important for inhibiting the expression of immune response genes, including those that are PAF1- and STAT2-independent. Nuclear localization of ZIKV and WNV NS5 were recently shown to be important for inhibition of IFN-I production $(50,51)$. NS5 nuclear localization could be a general mechanism by which flaviviruses inhibit immune gene expression, potentially through protein interactions with host gene expression machinery in the nucleus $(13,14)$. Interrogating these molecular mechanisms will be an important future direction in establishing the role of nuclear NS5.

\section{Conclusions}

In summary, we interrogate the role of PAF1C in DENV2 replication, regulating immune gene expression and NS5 antagonism of this process. By mapping the viral determinants of the NS5PAF1C protein interaction and creating NS5 mutants in which the NS5-PAF1C interaction is 
broken, we rescued the expression of PAF1-dependent immune response genes. These mutants also rescued the expression of immune response genes that are PAF1- and STAT2-independent. As such, NS5 antagonizes the expression of immune response genes using multiple mechanisms. Exploring PAF1- and STAT2-independent mechanisms in the future will reveal novel insights into the role of nuclear NS5.

\section{Materials and Methods}

Cells and viruses. A549 (ATCC), HEK293T (gift of Sam Díaz-Muñoz) and Vero (ATCC) cell lines were maintained in Dulbecco's modified Eagle's medium (DMEM, Gibco ThermoFisher) supplemented with $10 \%$ fetal bovine serum (FBS, Gibco ThermoFisher) at $37^{\circ} \mathrm{C}$. Cells were tested for mycoplasma monthly by PCR. Clonal A549 cells knockout (KO) for STAT2 and PAF1 were generated by CRISPR/Cas9. The CRISPR guide RNA sequences are from the GeCKO v2 Human library (52). The gRNA oligos were clones into the lentiCRISPRv2 puro plasmid (Addgene \#52961, (52)). The cloning product $(3.5 \mu \mathrm{g})$ were transfected using calcium phosphate protocol into 293T cells with lentiviral packaging plasmids including $1.8 \mu \mathrm{g}$ pMDLg/p-RRE, $1.25 \mu \mathrm{g}$ pCMVVSV-g and $1.5 \mu \mathrm{g}$ pRSV-Rev (53). After 36 hours lentivirus particles were collected and cell debris was removed by centrifugation and filtration through a $0.45 \mu \mathrm{m}$ filter. The resulting lentiviral stocks were used to transduce parental A549 cells. Transduced cells were selected for puromycin resistance (10 $\mu \mathrm{g} / \mathrm{ml}$, ThermoFisher). PAF1 KO generation required a clonal selection by limiting dilution. PAF1 and STAT2 KO were verified by Sanger sequencing and immunoblotting using antibodies against PAF1 (1:1000, Bethyl Labs, A300-173A) or STAT2 (1:200, Santa Cruz Biotechnology) (S1 Fig). A549 PAF1 and STAT2 rescue cells were created by cloning PAF1 and STAT2 cDNA into pLenti6 plasmid (Addgene \#89766, 54). Lentiviral packaging was performed as described above. PAF1 and STAT2 KO cell lines were transduced and selected for Blasticidin resistance (12 $\mu \mathrm{g} / \mathrm{ml}$, InvivoGen). High titer stocks of DENV2 16681 strain were obtained by passage of a p0 stock in C6/36 cells (14).

Plasmids. All DENV2 truncated protein-encoding plasmids were generated in the pcDNA4_TO with a C-terminal 2xStrep II affinity tag using PCR amplification and Gibson assembly. Flaviviruses NS5-encoding plasmids were previously generated (DENV2, ZIKV, WNV) (14) or synthesized for Gibson assembly (TBEV, POWV, LGTV) (Genewiz, Gene Universal). DENV2 NS5 point mutations were generated using site-directed mutagenesis of pcDNA4_TO DENV2 WT 
NS5 plasmid. LentiCRISPRv2 Puro (Addgene \#52961, (52)) encoding Cas9 and gRNA targeting PAF1 and STAT2 were generated following the Sanjana et al. protocol. Plasmid encoding PAF1 was kind gift from Nevan Krogan. PAF1 and STAT2 (Addgene \#71451, 55) were inserted into pLenti6 via Gibson assembly. Primer sequences used for the generation of all our constructs are available in S6 Table.

Virus infection and plaque assay. PAF1 KO or rescue A549 cells were inoculated at a MOI of 0.1 and supernatants were removed at the indicate time and stored at $-80^{\circ} \mathrm{C}$. Samples were subjected to 10 -fold serial dilutions followed by incubation on a monolayer of Vero cells for two hours at $37^{\circ} \mathrm{C}$. Samples were removed and replaced with $0.8 \%$ methylcellulose (Sigma, MO512) in 1X DMEM supplemented with $2 \%$ FBS and $1 \%$ pen/strep. Plaque assays were incubated for 8 days at $37^{\circ} \mathrm{C}$, then fixed with $4 \%$ formaldehyde and stained with $0.23 \%$ crystal violet to visualize plaques.

Affinity purification and immunoblotting. HEK293T cells were seed with a density of $4 \times 10^{6}$ cells in $10 \mathrm{~cm}$ dishes and transfected with $5 \mu \mathrm{g}$ of Strep-tagged plasmid encoding the truncated NS5 or the Flaviviruses NS5 using Polyjet (1:3 ratio, Signagen). After 36 hours post-transfection lysis, affinity purification and washes were performed using buffer containing, Tris-HCL pH 7.4 50 $\mathrm{mM}, \mathrm{NaCl} 150 \mathrm{mM}$, EDTA $1 \mathrm{mM}$ and 0.5\% NP40, supplemented with protease inhibitor mini tablets and phosphatase inhibitor mini tablets (ThermoFisher Scientific). The REAP protocol (56) was used to separate nuclear and cytoplasmic fractions. All samples were treated similarly for the affinity purification. Pulldown of Strep-tagged protein was performed using Strep-tactin beads (IBA Lifescience) as previously described (14). Protein samples were resuspended in 4:1 NuPage:TCEP loading buffer, heated for $10 \mathrm{~min}$ at $95^{\circ} \mathrm{C}$. Both cell lysates and affinity purification fractions were separated by SDS-Page on $7.5 \%$ polyacrylamide gels. Proteins were transferred onto PDVF $0.2 \mu \mathrm{m}$ membrane (Hybond P, GE Healthcare). The membranes were blocked in 5\% non-fat milk in $0.1 \%$ TBS-Tween, then incubated overnight at $4^{\circ} \mathrm{C}$ or 2 hours at room temperature with primary antibody diluted in BSA $2.5 \%$ TBS-tween $0.1 \%$ blocking buffer. The primary antibodies were detected by secondary antibody anti-mouse or anti-rabbit conjugated to horseradish peroxidase (Southern Biotech, 1:10,000 dilution) for 1 hour at room temperature. The antibody-bound proteins were detected by incubating with Pierce ECL substrate or SuperSignal West Femto substrate (ThermoFisher) and visualize on a Amersham ${ }^{\text {TM }}$ Imager 600 system (GE Healthcare). The followed antibodies and dilutions were used: Strep 1:1000 (Qiagen, 34850), 
PAF1 1:1000 (Bethyl Labs \#A300-173A), CDC73 1:500 (GeneTex, GTX110280), LEO1 1:1000 (Bethyl Labs \#A300-173A), STAT2 1:200 (Santa-Cruz Biotechnology, sc-514193), GAPDH 1:1000 (Cell Signaling Technology, 14C10), Calnexin 1:1000 (Cell Signaling Technology, C5C9) Lamin A/C 1:1000 (Cell Signaling Technology, 4C11).

Confocal immunofluorescence microscopy. A549 cells were fixed with $4 \%$ paraformaldehyde 24 hours post transfection. Cells were permeabilized with $0.25 \%$ Triton X-100 for 5 min and blocked with $5 \%$ goat serum (Sigma) in PBS. Coverslips (\#1.5) were incubated for 1 hour with primary antibody followed by 1 hour with secondary antibody at room temperature. Nuclei were visualized with Hoechst (Invitrogen). Confocal microscopy was performed with confocal Olympus FV 1000 microscope using 60x/1.35 oil immersion objective. AlexaFluor 488, AlexaFluor 555 and Hoechst 33342 were detected using $488 \mathrm{~nm}, 543 \mathrm{~nm}$ and $405 \mathrm{~nm}$, respectively, all at 1:1000 dilution. For the microscopy we used the following antibodies: PAF1 1:500 (Atlas antibodies, HPA043637), Strep 1:1000 (Qiagen, 34850).

Transcriptomic sample preparation. Parental, PAF1 KO/PAF1 rescue, STAT2 KO/ STAT2 rescue A549 cells were plated at a density of $9 \times 10^{5}$ cells per well in 6 -well plates. Cells were stimulated with poly(l:C) at a final concentration of $2 \mu \mathrm{g} / \mathrm{mL}$ using $4 \mu \mathrm{L}$ Lipofectamine 2000 (Thermo Fisher). At three hours post-transfection, total cellular RNA was extracted using the RNeasy mini kit (Qiagen). For experiments involved GFP and NS5 expression, 2.5 and $5 \mu \mathrm{g}$ of plasmid DNA was transfected using a ratio of 1:2 plasmid:Lipofectamine 300036 hours before poly(l:C) stimulation. RNA samples were prepared with the QuantSeq3' mRNA-Seq Library kit for Illumina (Lexogen $\mathrm{GmbH}$ ) following standard protocol (57). The resulting cDNA libraries were analyzed with NextSeq 500 sequencing run with 4 million reads per sample.

RNA-seq data processing. Quality of all reads was evaluated using FastQC (v0.11.5). To remove the 3' read end poly-A stretches the reads were trimmed (bbduk, sourceforge.net/projects/bbmap/). All remaining sequences were mapped against the human reference genome build 38 with STAR (v2.5.2b) (58). HTseq (v0.6.1) was used to count all reads for each gene and set up a read count table (59). Differential gene expression analyses were performed using the DESeq2 Bioconductor package (v1.30.1) (60). The default "apelgm" shrinkage (v1.28.0) (61) set up was used for our analysis. Gene-set enrichment analysis (GSEA) 
was performed with the fgsea Bioconductor package (62), using Reactome gene sets downloaded from the Molecular Signatures Database (63).

Quantitative reverse transcription-PCR (qRT-PCR). Total RNA was isolated using RNeasy mini kit (Qiagen) column purification. cDNA was synthesized with iScript kit (BioRad). Real-time PCR was performed with iTaq SYBR Green premix (BioRad) and data were collected with LightCycler® 480 (Roche). All Ct values were normalized to the expression values of GAPDH RNA and gene expression quantification were performed by the $2^{-\Delta \Delta C t}$ method (64). The primers sequences are provided in S6 Table.

Statistical analysis. For plaque assay of virus replication data, $\mathrm{p}$ values were calculated using a paired, one-tailed Student's t-test. For differential gene expression analysis, significant changes in specific genes ( $p$ adj $<0.05$ ) were identified after adjusting for false discovery rate using the Benjamini Hochberg method. For GSEA, all genes were included in the random walk, with 10,000 iterations occurring for gene-sets of size 50 to 1000 . Significant changes in the expression of groups of genes were calculated using a Wilcoxon signed rank test with Bonferroni correction for multiple testing for paired samples and Wilcoxon rank test with Bonferroni correction for multiple testing for unpaired samples. Overlap between gene sets was tested using a Fishers exact test.

\section{Acknowledgements}

We would like to thank members of the Shah lab and Marty Privalsky for fruitful discussions and critical feedback. Funding was provided by University of California, Davis and the W. M. Keck foundation. MJP was supported by the Philippe Foundation Inc. The sequencing was carried out at the DNA Technologies and Expression Analysis Cores at the UC Davis Genome Center, supported by NIH Shared Instrumentation Grant 1S10OD010786-01. This work is dedicated to the memory of Marty Privalsky, a wonderful colleague, scientist and friend.

\section{Author Contributions}

MJP and PSS conceived of work and designed experiments. MJP, MWK and AAN performed experiments. MJP, MWK and PSS performed bioinformatic analysis. MJP, MWK, AAN and PSS wrote manuscript. PSS secured funding for work. All authors read and approved the final manuscript. 


\section{References}

1. Bhatt S, Gething PW, Brady OJ, Messina JP, Farlow AW, Moyes CL, et al. The global distribution and burden of dengue. Nature. 2013 Apr;496(7446):504-7.

2. Tan B-H, Fu J, Sugrue RJ, Yap E-H, Chan Y-C, Tan YH. Recombinant Dengue Type 1 Virus NS5 Protein Expressed in Escherichia coli Exhibits RNA-Dependent RNA Polymerase Activity. Virology. 1996 Feb 15;216(2):317-25.

3. Egloff M-P, Benarroch D, Selisko B, Romette J-L, Canard B. An RNA cap (nucleoside-2'-O)-methyltransferase in the flavivirus RNA polymerase NS5: crystal structure and functional characterization. EMBO J. 2002 Jun 3;21(11):2757-68.

4. Dong H, Chang DC, Xie X, Toh YX, Chung KY, Zou G, et al. Biochemical and genetic characterization of dengue virus methyltransferase. Virology. 2010 Sep 30;405(2):568-78.

5. Ray D, Shah A, Tilgner M, Guo Y, Zhao Y, Dong H, et al. West Nile Virus 5'-Cap Structure Is Formed by Sequential Guanine N-7 and Ribose 2'-O Methylations by Nonstructural Protein 5. J Virol. 2006 Sep 1;80(17):8362-70.

6. Ashour J, Laurent-Rolle M, Shi P-Y, García-Sastre A. NS5 of dengue virus mediates STAT2 binding and degradation. J Virol. 2009 Jun;83(11):5408-18.

7. Kapoor M, Zhang L, Mohan PM, Padmanabhan R. Synthesis and characterization of an infectious dengue virus type-2 RNA genome (New Guinea C strain). Gene. 1995 Sep 11;162(2):175-80.

8. Kumar A, Bühler S, Selisko B, Davidson A, Mulder K, Canard B, et al. Nuclear localization of Dengue virus non-structural protein 5 does not strictly correlate with efficient viral RNA replication and inhibition of type I interferon signaling. J Virol. 2013 Feb 13;JVI.03083-12.

9. Tay MYF, Smith K, Ng IHW, Chan KWK, Zhao Y, Ooi EE, et al. The C-terminal 18 Amino Acid Region of Dengue Virus NS5 Regulates its Subcellular Localization and Contains a Conserved Arginine Residue Essential for Infectious Virus Production. PLOS Pathog. 2016 Sep 13;12(9):e1005886.

10. Pryor MJ, Rawlinson SM, Butcher RE, Barton CL, Waterhouse TA, Vasudevan SG, et al. Nuclear localization of dengue virus nonstructural protein 5 through its importin alpha/betarecognized nuclear localization sequences is integral to viral infection. Traffic Cph Den. 2007 Jul;8(7):795-807. 
11. Hannemann H, Sung P-Y, Chiu H-C, Yousuf A, Bird J, Lim SP, et al. Serotype Specific Differences in Dengue Virus Non-Structural Protein 5 Nuclear Localization. J Biol Chem. 2013 Jun 13;jbc.M113.481382.

12. Khadka S, Vangeloff AD, Zhang C, Siddavatam P, Heaton NS, Wang L, et al. A Physical Interaction Network of Dengue Virus and Human Proteins*. Mol Cell Proteomics. 2011 Dec 1;10(12):M111.012187.

13. Maio FAD, Risso G, Iglesias NG, Shah P, Pozzi B, Gebhard LG, et al. The Dengue Virus NS5 Protein Intrudes in the Cellular Spliceosome and Modulates Splicing. PLOS Pathog. 2016 Aug 30;12(8):e1005841.

14. Shah PS, Link N, Jang GM, Sharp PP, Zhu T, Swaney DL, et al. Comparative FlavivirusHost Protein Interaction Mapping Reveals Mechanisms of Dengue and Zika Virus Pathogenesis. Cell. 2018 Dec 13;175(7):1931-1945.e18.

15. Marazzi I, Ho JSY, Kim J, Manicassamy B, Dewell S, Albrecht RA, et al. Suppression of the antiviral response by an influenza histone mimic. Nature. 2012 Mar;483(7390):428-33.

16. Parnas O, Jovanovic M, Eisenhaure TM, Herbst RH, Dixit A, Ye CJ, et al. A Genome-wide CRISPR Screen in Primary Immune Cells to Dissect Regulatory Networks. Cell. 2015 Jul 30;162(3):675-86.

17. Chu X, Qin X, Xu H, Li L, Wang Z, Li F, et al. Structural insights into Paf1 complex assembly and histone binding. Nucleic Acids Res. 2013 Dec 1;41(22):10619-29.

18. Olagnier D, Scholte FEM, Chiang C, Albulescu IC, Nichols C, He Z, et al. Inhibition of Dengue and Chikungunya Virus Infections by RIG-I-Mediated Type I Interferon-Independent Stimulation of the Innate Antiviral Response. J Virol. 2014 Apr 15;88(8):4180-94.

19. Kato H, Takeuchi O, Mikamo-Satoh E, Hirai R, Kawai T, Matsushita K, et al. Lengthdependent recognition of double-stranded ribonucleic acids by retinoic acid-inducible geneI and melanoma differentiation-associated gene 5. J Exp Med. 2008 Jun 30;205(7):160110.

20. Okahira S, Nishikawa F, Nishikawa S, Akazawa T, Seya T, Matsumoto M. Interferon- $\beta$ Induction Through Toll-Like Receptor 3 Depends on Double-Stranded RNA Structure. DNA Cell Biol. 2005 Oct;24(10):614-23.

21. Matsumoto $M$, Seya T. TLR3: Interferon induction by double-stranded RNA including poly(I:C). Adv Drug Deliv Rev. 2008 Apr 29;60(7):805-12. 
22. Mootha VK, Lindgren CM, Eriksson K-F, Subramanian A, Sihag S, Lehar J, et al. PGC-1aresponsive genes involved in oxidative phosphorylation are coordinately downregulated in human diabetes. Nat Genet. 2003 Jul;34(3):267-73.

23. Subramanian A, Tamayo P, Mootha VK, Mukherjee S, Ebert BL, Gillette MA, et al. Gene set enrichment analysis: A knowledge-based approach for interpreting genome-wide expression profiles. Proc Natl Acad Sci. 2005 Oct 25;102(43):15545-50.

24. Loo Y-M, Gale M. Immune Signaling by RIG-I-like Receptors. Immunity. 2011 May 27;34(5):680-92.

25. Nasirudeen AMA, Wong HH, Thien P, Xu S, Lam K-P, Liu DX. RIG-I, MDA5 and TLR3 Synergistically Play an Important Role in Restriction of Dengue Virus Infection. PLoS Negl Trop Dis. 2011 Jan 4;5(1):e926.

26. Qin C-F, Zhao H, Liu Z-Y, Jiang T, Deng Y-Q, Yu X-D, et al. Retinoic acid inducible gene-I and melanoma differentiation-associated gene 5 are induced but not essential for dengue virus induced type I interferon response. Mol Biol Rep. 2011 Aug;38(6):3867-73.

27. Chazal M, Beauclair G, Gracias S, Najburg V, Simon-Lorière E, Tangy F, et al. RIG-I Recognizes the 5' Region of Dengue and Zika Virus Genomes. Cell Rep. 2018 Jul 10;24(2):320-8.

28. Chan YK, Gack MU. A phosphomimetic-based mechanism of dengue virus to antagonize innate immunity. Nat Immunol. 2016 May;17(5):523-30.

29. Sprokholt JK, Kaptein TM, Hamme JL van, Overmars RJ, Gringhuis SI, Geijtenbeek TBH. RIG-I-like receptor activation by dengue virus drives follicular $\mathrm{T}$ helper cell formation and antibody production. PLOS Pathog. 2017 Nov 29;13(11):e1006738.

30. Chen K, Liu J, Cao X. Regulation of type I interferon signaling in immunity and inflammation: A comprehensive review. J Autoimmun. 2017 Sep 1;83.

31. Marceau CD, Puschnik AS, Majzoub K, Ooi YS, Brewer SM, Fuchs G, et al. Genetic dissection of Flaviviridae host factors through genome-scale CRISPR screens. Nature. 2016 Jul;535(7610):159-63.

32. Richardson RB, Ohlson MB, Eitson JL, Kumar A, McDougal MB, Boys IN, et al. A CRISPR screen identifies IFI6 as an ER-resident interferon effector that blocks flavivirus replication. Nat Microbiol. 2018 Nov;3(11):1214-23.

33. Zhang R, Miner JJ, Gorman MJ, Rausch K, Ramage H, White JP, et al. A CRISPR screen defines a signal peptide processing pathway required by flaviviruses. Nature. 2016 Jul;535(7610):164-8. 
34. Savidis G, McDougall WM, Meraner P, Perreira JM, Portmann JM, Trincucci G, et al. Identification of Zika Virus and Dengue Virus Dependency Factors using Functional Genomics. Cell Rep. 2016 Jun 28;16(1):232-46.

35. Li Y, Muffat J, Javed AO, Keys HR, Lungjangwa T, Bosch I, et al. Genome-wide CRISPR screen for Zika virus resistance in human neural cells. Proc Natl Acad Sci. 2019 May 7;116(19):9527-32.

36. Krishnan MN, Ng A, Sukumaran B, Gilfoy FD, Uchil PD, Sultana H, et al. RNA interference screen for human genes associated with West Nile virus infection. Nature. 2008 Sep;455(7210):242-5.

37. Miller S, Sparacio S, Bartenschlager R. Subcellular Localization and Membrane Topology of the Dengue Virus Type 2 Non-structural Protein 4B*. J Biol Chem. 2006 Mar 31;281(13):8854-63.

38. Hsu PL, Li H, Lau H-T, Leonen C, Dhall A, Ong S-E, et al. Crystal Structure of the COMPASS H3K4 Methyltransferase Catalytic Module. Cell. 2018 Aug 23;174(5):1106-1116.e9.

39. Krogan NJ, Dover J, Wood A, Schneider J, Heidt J, Boateng MA, et al. The Paf1 complex is required for histone $\mathrm{H} 3$ methylation by COMPASS and Dot1p: linking transcriptional elongation to histone methylation. Mol Cell. 2003 Mar;11(3):721-9.

40. Kovanich D, Saisawang C, Sittipaisankul P, Ramphan S, Kalpongnukul N, Somparn P, et al. Analysis of the Zika and Japanese Encephalitis Virus NS5 Interactomes. J Proteome Res. 2019 Aug 2;18(8):3203-18.

41. Li X-D, Shan C, Deng C-L, Ye H-Q, Shi P-Y, Yuan Z-M, et al. The Interface between Methyltransferase and Polymerase of NS5 Is Essential for Flavivirus Replication. PLoS Negl Trop Dis. 2014 May 22;8(5):e2891.

42. Lu G, Gong P. Crystal Structure of the Full-Length Japanese Encephalitis Virus NS5 Reveals a Conserved Methyltransferase-Polymerase Interface. PLOS Pathog. 2013 Aug 8;9(8):e1003549.

43. Guo X, Wang H, Li Y, Leng X, Huang W, Ma Y, et al. Transfection reagent Lipofectamine triggers type I interferon signaling activation in macrophages. Immunol Cell Biol. 2019;97(1):92-6.

44. Chen FX, Woodfin AR, Gardini A, Rickels RA, Marshall SA, Smith ER, et al. PAF1, a Molecular Regulator of Promoter-Proximal Pausing by RNA Polymerase II. Cell. 2015 Aug 27;162(5):1003-15. 
45. Yu M, Yang W, Ni T, Tang Z, Nakadai T, Zhu J, et al. RNA polymerase II-associated factor 1 regulates the release and phosphorylation of paused RNA polymerase II. Science. 2015 Dec 11;350(6266):1383-6.

46. Ding L, Paszkowski-Rogacz M, Mircetic J, Chakraborty D, Buchholz F. The Paf1 complex positively regulates enhancer activity in mouse embryonic stem cells. Life Sci Alliance. 2021 Mar;4(3):e202000792.

47. Best SM. The Many Faces of the Flavivirus NS5 Protein in Antagonism of Type I Interferon Signaling. J Virol. 2017 Jan 18; 91(3).

48. Lubick KJ, Robertson SJ, McNally KL, Freedman BA, Rasmussen AL, Taylor RT, et al. Flavivirus Antagonism of Type I Interferon Signaling Reveals Prolidase as a Regulator of IFNAR1 Surface Expression. Cell Host Microbe. 2015 Jul 8;18(1):61-74.

49. Grant A, Ponia SS, Tripathi S, Balasubramaniam V, Miorin L, Sourisseau M, et al. Zika Virus Targets Human STAT2 to Inhibit Type I Interferon Signaling. Cell Host Microbe. 2016 Jun 8;19(6):882-90.

50. Zhao Z, Tao M, Han W, Fan Z, Imran M, Cao S, et al. Nuclear localization of Zika virus NS5 contributes to suppression of type I interferon production and response. J Gen Virol. 2019 Dec 20.

51. Lopez-Denman AJ, Tuipulotu DE, Ross JB, Trenerry AM, White PA, Mackenzie JM. Nuclear localisation of West Nile virus NS5 protein modulates host gene expression. Virology. 2021 Apr 9;559:131-44.

52. Sanjana NE, Shalem O, Zhang F. Improved vectors and genome-wide libraries for CRISPR screening. Nat Methods. 2014 Aug;11(8):783-4.

53. Yu JH, Schaffer DV. Selection of Novel Vesicular Stomatitis Virus Glycoprotein Variants from a Peptide Insertion Library for Enhanced Purification of Retroviral and Lentiviral Vectors. J Virol. 2006 Apr 1;80(7):3285-92.

54. Pemble H, Kumar P, van Haren J, Wittmann T. GSK3-mediated CLASP2 phosphorylation modulates kinetochore dynamics. J Cell Sci. 2017 Apr 15;130(8):1404-12.

55. Cheon H, Holvey-Bates EG, Schoggins JW, Forster S, Hertzog P, Imanaka N, et al. IFN $\beta$ dependent increases in STAT1, STAT2, and IRF9 mediate resistance to viruses and DNA damage. EMBO J. 2013 Oct 16;32(20):2751-63.

56. Suzuki K, Bose P, Leong-Quong RY, Fujita DJ, Riabowol K. REAP: A two minute cell fractionation method. BMC Res Notes. 2010 Nov 10;3(1):294. 
57. Moll P, Ante M, Seitz A, Reda T. QuantSeq 3' mRNA sequencing for RNA quantification. Nat Methods. 2014 Dec;11(12):i-iii.

58. Dobin A, Davis CA, Schlesinger F, Drenkow J, Zaleski C, Jha S, et al. STAR: ultrafast universal RNA-seq aligner. Bioinformatics. 2013 Jan;29(1):15-21.

59. Anders S, Pyl PT, Huber W. HTSeq--a Python framework to work with high-throughput sequencing data. Bioinformatics. 2015 Jan 15;31(2):166-9.

60. Love MI, Huber W, Anders S. Moderated estimation of fold change and dispersion for RNAseq data with DESeq2. Genome Biol. 2014 Dec;15(12):550.

61. Zhu A, Ibrahim JG, Love MI. Heavy-tailed prior distributions for sequence count data: removing the noise and preserving large differences. Bioinformatics. 2019 Jun 1;35(12):2084-92.

62. Korotkevich G, Sukhov V, Budin N, Shpak B, Artyomov MN, Sergushichev A. Fast gene set enrichment analysis. bioRxiv. 2021 Feb 1;060012.

63. Liberzon A, Subramanian A, Pinchback R, Thorvaldsdottir H, Tamayo P, Mesirov JP. Molecular signatures database (MSigDB) 3.0. Bioinformatics. 2011 Jun 15;27(12):1739-40.

64. Livak KJ, Schmittgen TD. Analysis of Relative Gene Expression Data Using Real-Time Quantitative PCR and the 2- $\triangle \Delta C T$ Method. Methods. 2001 Dec;25(4):402-8. 


\section{Supplementary Figures}
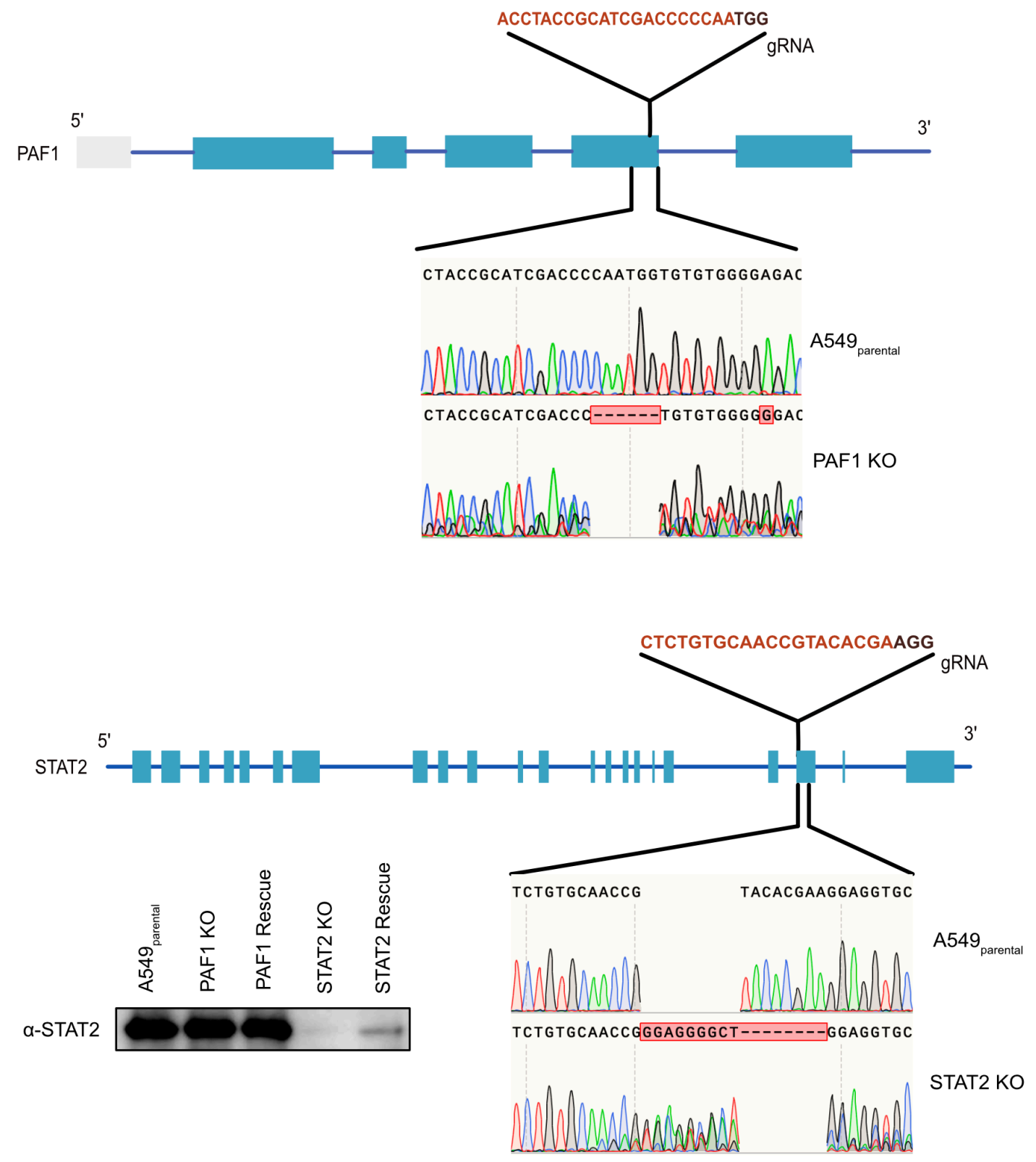

S1 Fig. Generation of PAF1 and STAT2 KO A549 cells

(A) PAF1 gRNA target region and Sanger sequencing of the CRISPR/Cas9 induced deletion. (B) STAT2 gRNA target region and Sanger sequencing of the CRISPR/Cas9 induced deletion. Immunoblotting was performed on protein extracted from parental A549, PAF1 KO/rescue and STAT2 KO/rescue. Immunostaining with STAT2 antibody showed the protein depletion for the STAT2 KO cells. STAT2 rescue data showed the restoration of the STAT2 detection. 
A GSEA of flavivirus host dependency factors
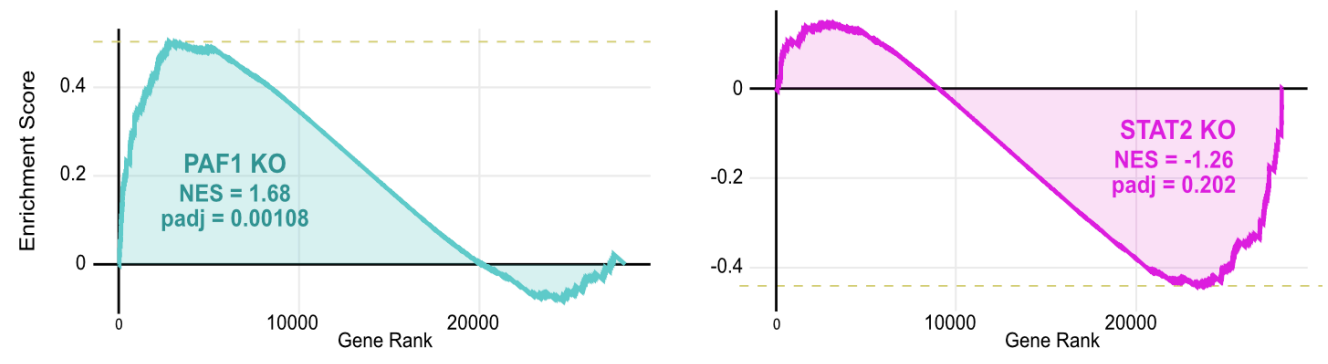

B Leading edge of flavivirus host dependency factor GSEA

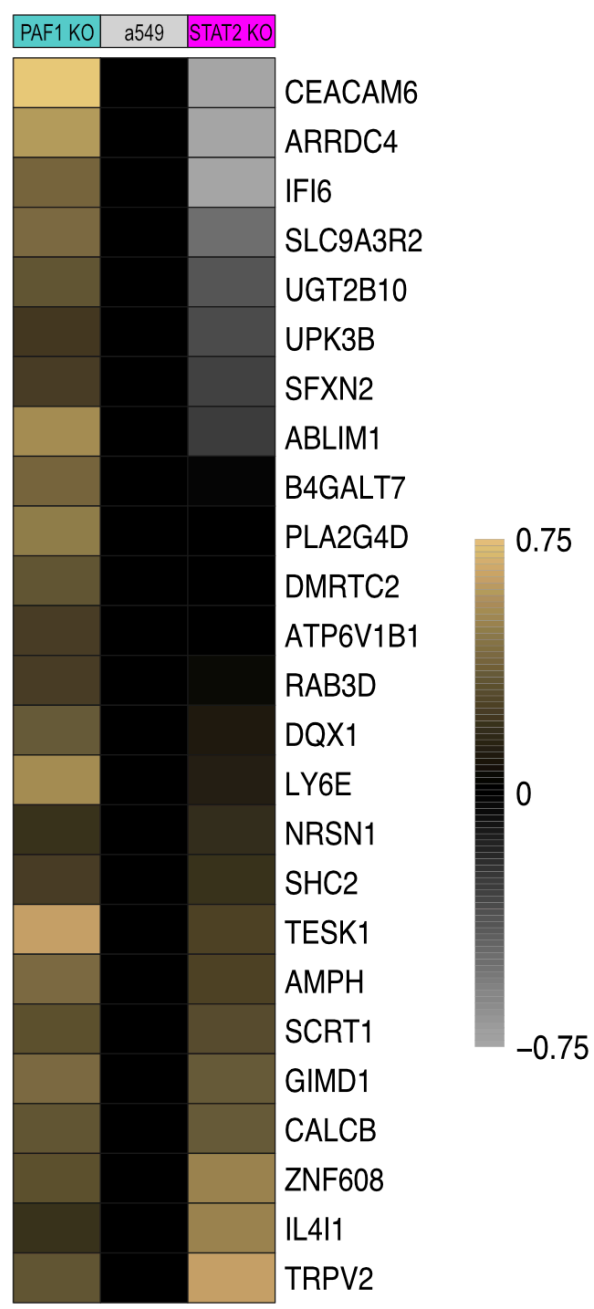

\section{S2 Fig. Impact of PAF1 KO on flavivirus host dependency factors}

(A) GSEA was performed using list of flavivirus host dependency genes. (B) Leading edge of flavivirus host dependency factors from GSEA. Heatmap represents log2 fold change relative to parental A549 following poly(l:C) treatment. 


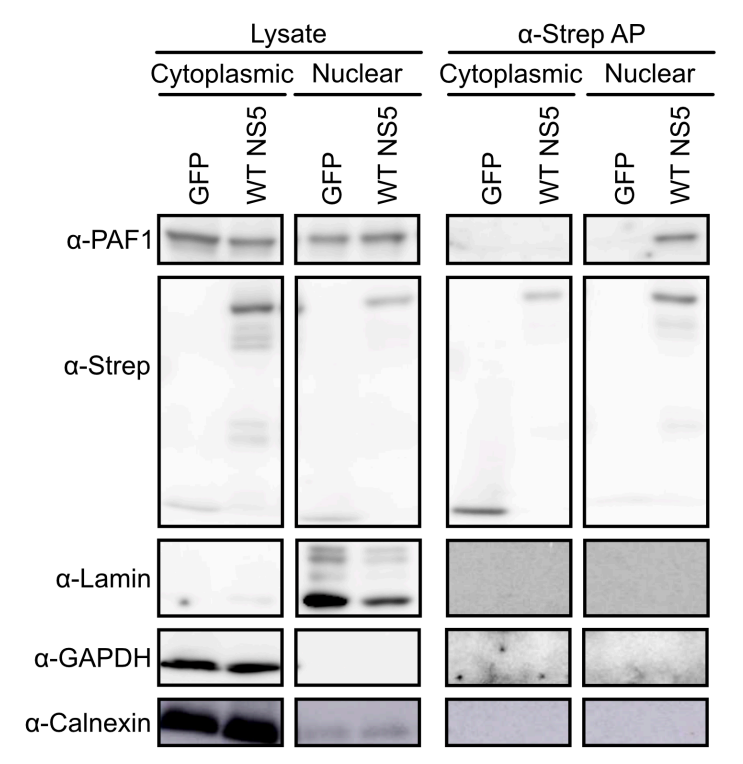

\section{S3 Fig. Subcellular localization of the NS5-PAF1 protein interaction}

Following nuclear/cytoplasmic fractionation, NS5 and GFP were subjected to affinity purification and immunoblot. Lamin and Calnexin served as controls for fraction purity. 

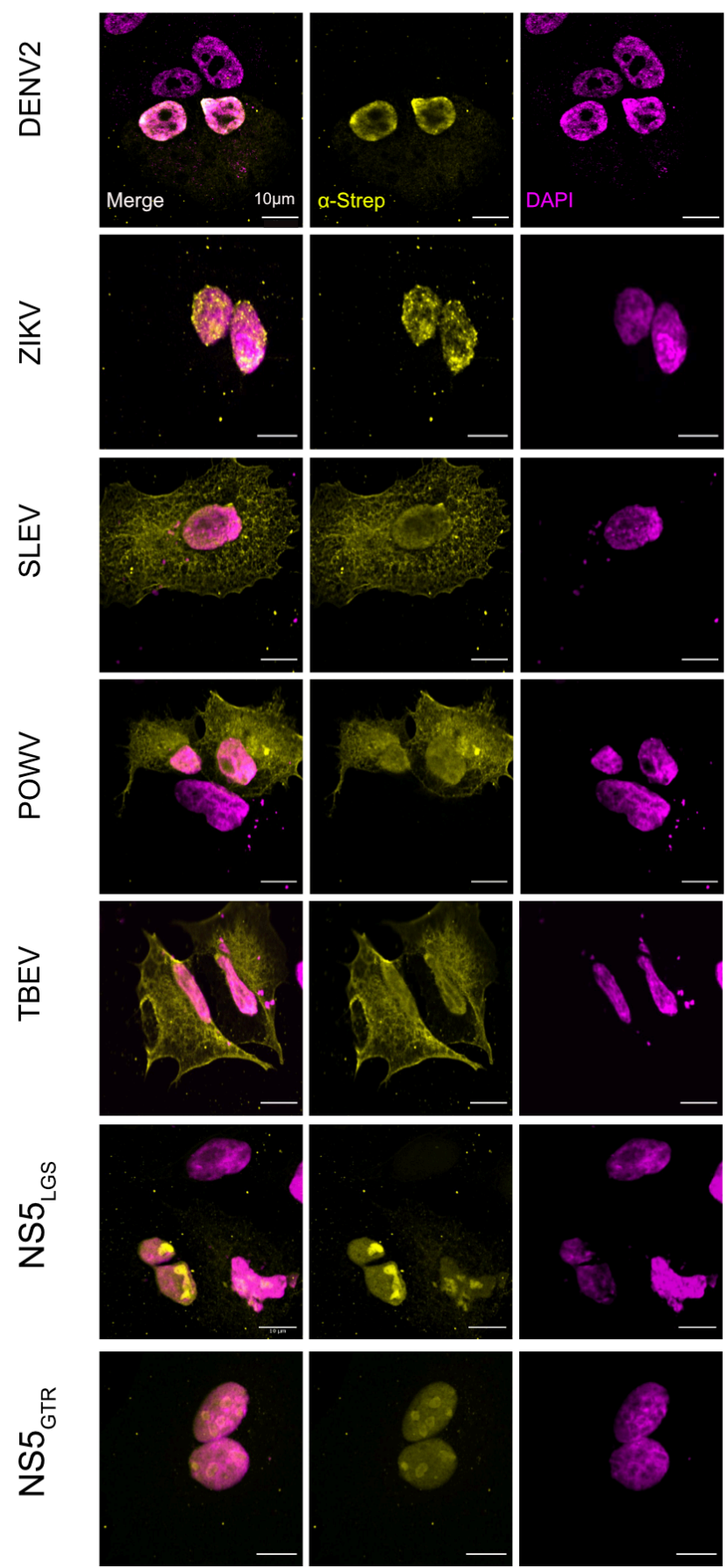

S4 Fig. Subcellular localization of flavivirus NS5s and mutant NS5s by confocal microscopy

Subcellular localization of 2xStrep II tagged flavivirus NS5s, NS5 LGS and NS5GTR (yellow) was determined by immunostaining and confocal microscopy. Nuclei were stained with Hoechst (magenta). Scale bar represents $10 \mu \mathrm{m}$. 
A Overall poly(l:C) induction

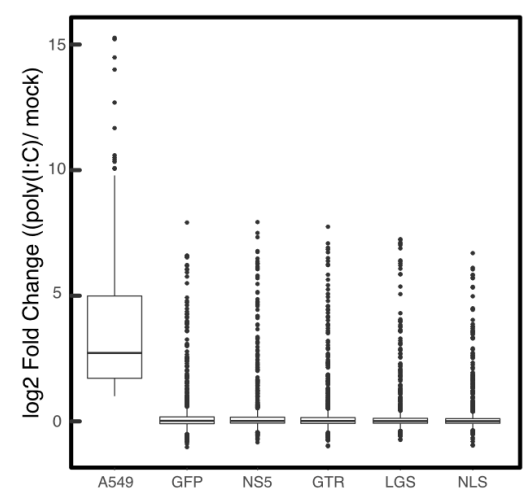

B Correlations between DNA transfection and poly $(\mathrm{I}: \mathrm{C})$ stimulation
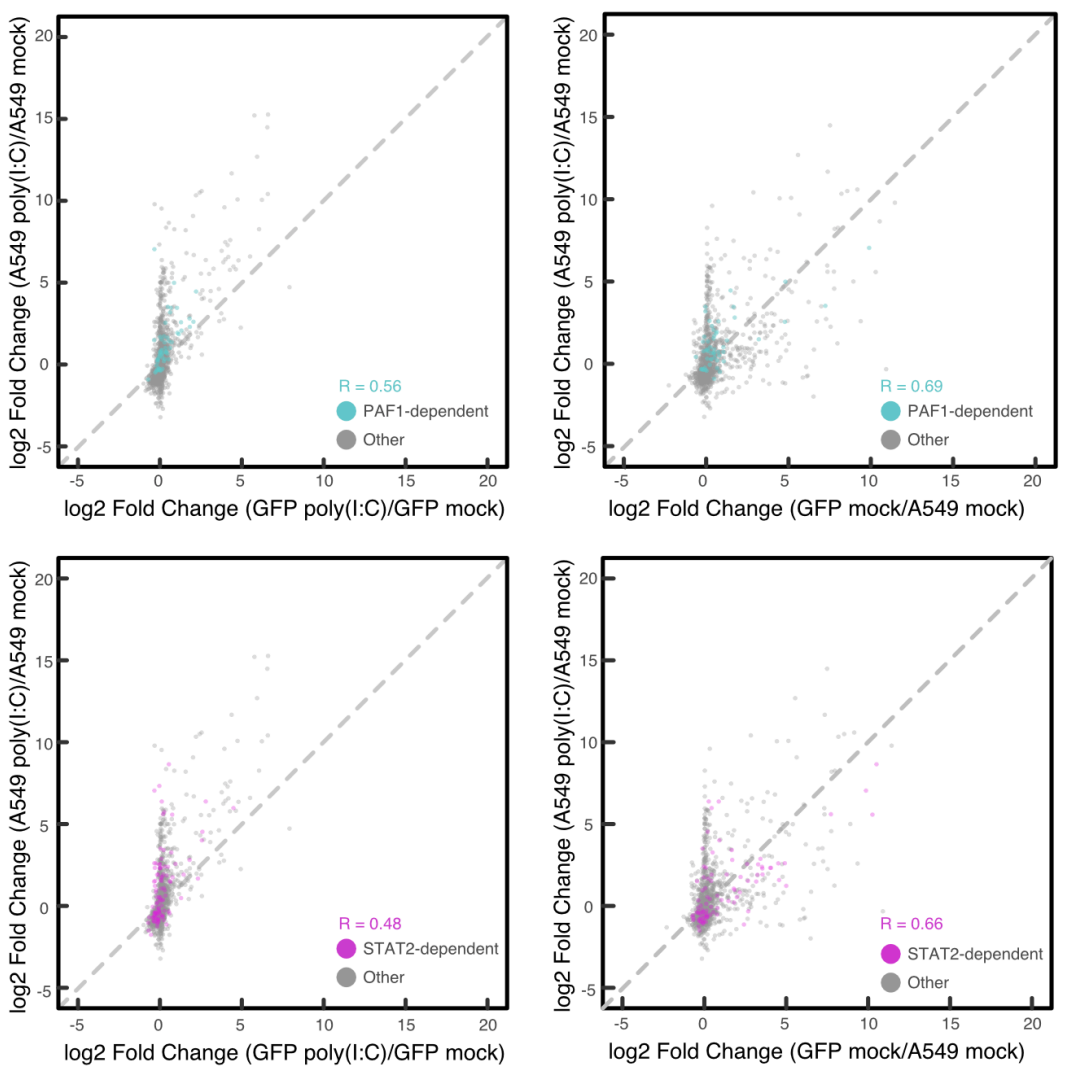

S5 Fig. Characterization of immune response in DNA-transfected A549 cells

(A) Changes in gene expression caused by poly(I:C) treatment are shown for the subset of immune response genes (GO:0006955) significantly upregulated for poly(l:C)-treated parental A549 cells relative to mock-treated A549 cells (log2 fold change $>0.5$, padj < 0.05 ). (B) Pearson's correlation coefficients were calculated for differential gene expression comparing DNA transfection and poly(l:C) stimulation. 

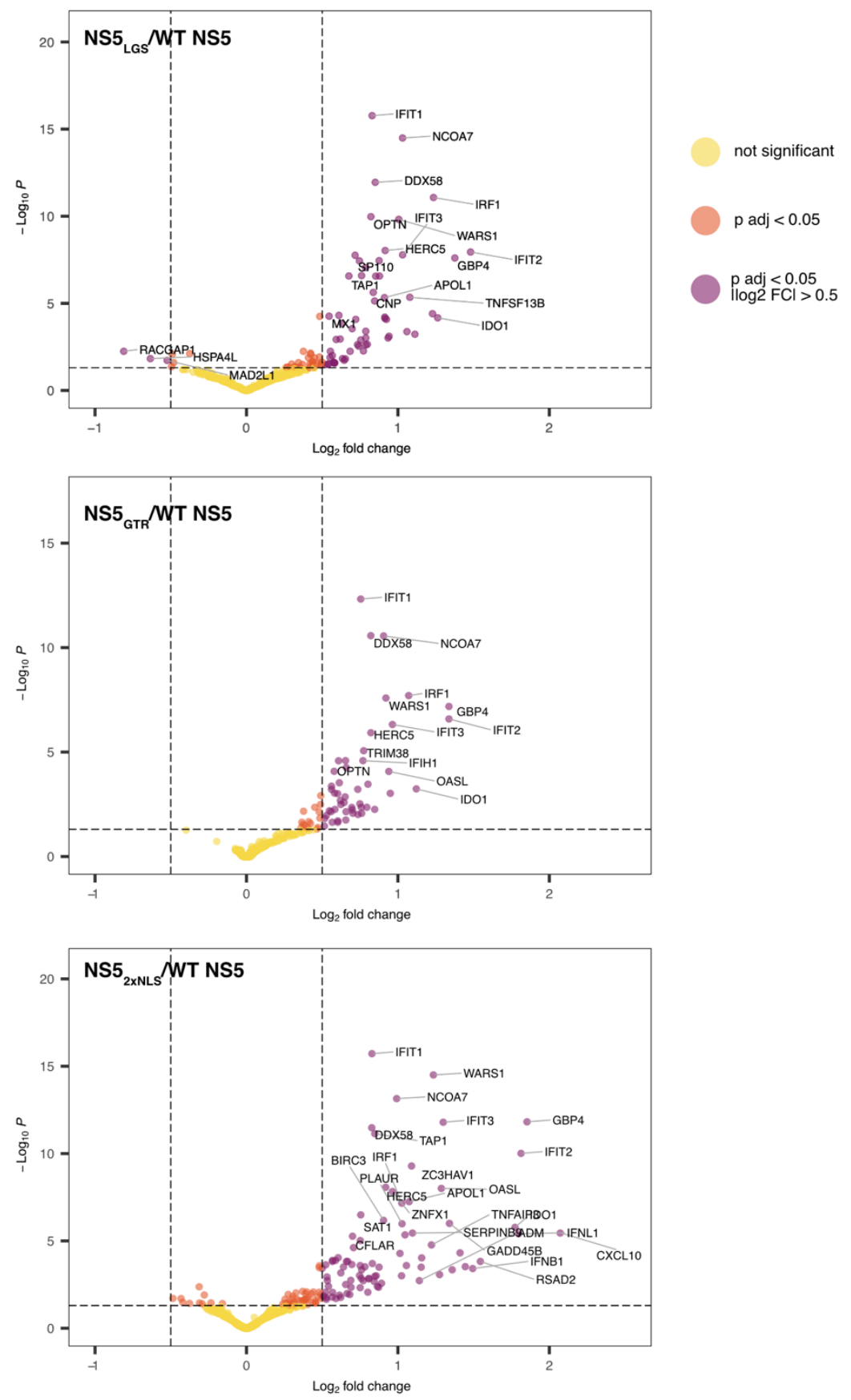

\section{S6 Fig. Gene expression analysis of NS5 mutants}

Relative change in gene expression was plotted as log2 fold change versus adjusted $p$ value to identify general trends for (A) NS5LGS, (B) NS5GTR and (C) NS5 $5_{2 \times N L S}$. Genes with significant increases (log2 fold change $>0.5$, padj $<0.05$ ) for $N S 5_{2 \times N L S}$ were used for heatmap analysis in Fig $6 C$. 
qRT-PCR validation
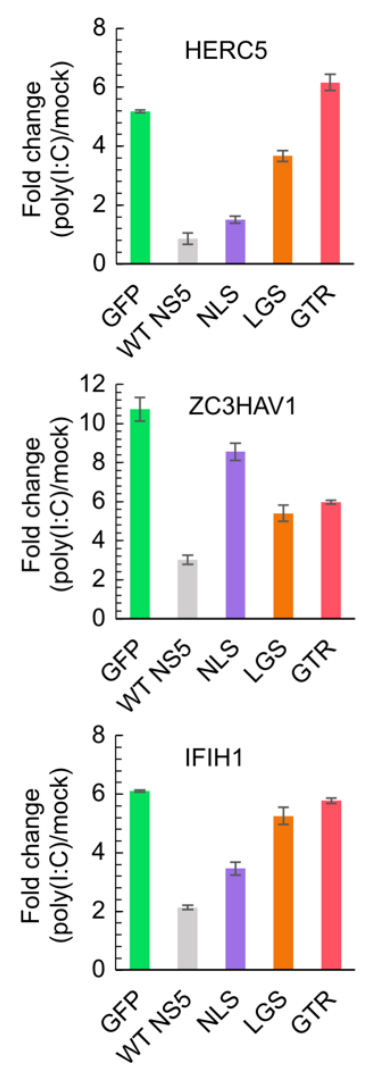

S7 Fig. qRT-PCR analysis of PAF1-dependent genes rescued by NS5 mutants

qRT-PCR was performed on PAF1-dependent immune response genes from Fig 7A. Fold changes were calculated using the $\triangle \Delta \mathrm{Ct}$ method and normalized to GAPDH as the housekeeping gene. GFP transfection was used as a positive control for poly(l:C) induction. 